\title{
INVITED SURVEY PAPER Nonlinear Shannon Limit in Optical Fiber Transmission System
}

\author{
Akihiro MARUTA ${ }^{\dagger a)}$, Senior Member
}

\begin{abstract}
SUMMARY The remaining issues in optical transmission technology are the degradation of optical signal to noise power ratio due to amplifier noise and the distortions due to optical nonlinear effects in a fiber. Therefore in addition to the Shannon limit, practical channel capacity is believed to be restricted by the nonlinear Shannon limit. The nonlinear Shannon limit has been derived under the assumption that the received signal points on the constellation map deviated by optical amplifier noise and nonlinear interference noise are symmetrically distributed around the ideal signal point and the sum of the noises are regarded as white Gaussian noise. The nonlinear Shannon limit is considered as a kind of theoretical limitation. However it is doubtful that its derivation process and applicable range have been understood well. In this paper, some fundamental papers on the nonlinear Shannon limit are reviewed to better understanding its meaning and applicable range.

key words: optical fiber communication, nonlinear Shannon limit, Gaussian noise model, four wave mixing
\end{abstract}

\section{Introduction}

The Shannon limit [1] which represents the theoretical upper limit of the channel capacity is the most important and most famous expression in communication engineering. The spectral efficiency $C / W[\mathrm{bit} / \mathrm{s} / \mathrm{Hz}]$ is defined as the channel capacity $C[\mathrm{bit} / \mathrm{s}]$ per unit bandwidth under the influence of white Gaussian noise. The expression of the Shannon limit is given by

$$
\frac{C}{W}=\log _{2}(1+\mathrm{SNR}),
$$

where SNR is the signal-to-noise ratio and $W[\mathrm{~Hz}]$ represents the bandwidth.

The information is transmitted by modulating amplitude, frequency and/or phase of lightwave following the temporal variation of information data in optical fiber communication system. In conventional systems, binary digital transmission is carried out by assigning the binary data, ' 0 ' and ' 1 ', to the status that the light is on and off, respectively. Recently, research and development on multi-level optical transmission in which amplitude and phase of lightwave are modulated have been actively carried out and their practical implementation has already begun [2] by the development of coherent optical communication technology [3], [4]. Optical transmission technologies, which can overcome fiber loss by

\footnotetext{
Manuscript received October 1, 2016.

Manuscript revised April 18, 2017.

Manuscript publicized May 30, 2017.

$\dagger$ The author is with the Osaka University, Suita-shi, 565-0871 Japan.

a)E-mail: maruta@comm.eng.osaka-u.ac.jp

DOI: $10.1587 /$ transcom.2016EBI0003
}

optical amplification and overcome linear distortions due to group velocity dispersion and polarization mode dispersion by coherent detection assisted by digital signal processing, have remaining problems caused by the degradation of optical signal-to-noise power ratio (OSNR) and the distortions due to optical nonlinear effects in a fiber. Therefore in addition to the Shannon limit, practical channel capacity is believed to be limited by nonlinear Shannon limit [5]-[17]. The nonlinear Shannon limit has been derived under the assumption that the received signal points on the constellation map deviated by optical amplifier noise and nonlinear interference noise is assumed to be symmetrically distributed around the ideal signal point and the sum of the noises are regarded as white Gaussian noise. The nonlinear Shannon limit is considered as a kind of theoretical limitation. However it is doubtful that its derivation process and applicable range have been understood well.

In this paper, some fundamental papers [5]-[8] on the nonlinear Shannon limit are reviewed to better understanding its meanings and applicable range. In Sect. 2, we derive a model equation which describes a behavior of lightwave propagation in a fiber. In Sect. 3, the deductive derivation of the nonlinear Shannon limit starting from the model equation is presented. In Sect. 4, the application of the derived expressions to a practical system evaluation and design are discussed. Finally, Sect. 5 is devoted to conclude the discussion.

\section{Lightwave Propagation in a Fiber}

A model equation which describes lightwave propagation in a fiber is reviewed in this section [18].

\subsection{Lightwave Propagation in a Linear Fiber}

We consider an electric field of a linearly polarized (LP) mode propagating in a single mode fiber which can be treated with weakly guiding approximation. By setting the $z$-axis to the fiber axis, we assume that the electric field $\boldsymbol{E}\left(\boldsymbol{r}_{\perp}, z, t\right)$ of lightwave in a fiber can be expressed as

$$
\begin{aligned}
& \boldsymbol{E}\left(\boldsymbol{r}_{\perp}, z, t\right) \\
& \quad=\boldsymbol{i} \operatorname{Re}\left[E(z, t) \phi\left(\boldsymbol{r}_{\perp}\right) \exp \left\{i\left(\omega_{0} t-\beta_{0} z\right)\right\}\right],
\end{aligned}
$$

where $\operatorname{Re}[*]$ represents the real part of $[*] . \quad \boldsymbol{r}_{\perp}$ is the coordinate on the fiber's cross section which is perpendicular to the $z$-axis. $t[\mathrm{~s}]$ expresses the time. $i$ represents a unit 
vector towards the direction of polarization, i.e., the direction of the electric field, and it is perpendicular to the $z$-axis. $E(z, t)$ expresses a complex amplitude of the envelope of the electric field and its absolute value represents the pulse shape. $\phi\left(\boldsymbol{r}_{\perp}\right)$ represents the modal function of the fundamental mode ( $\mathrm{LP}_{01}$ mode). $i=\sqrt{-1}$ is the imaginary unit. $\omega_{0}[\mathrm{rad} / \mathrm{s}]$ represents the angular frequency of the carrier. $\beta_{0}[\mathrm{rad} / \mathrm{m}]$ represents the propagation constant of the fundamental mode at $\omega_{0}$.

Next, we calculate the Fourier transform of the optical pulse, $E(z, t)$, with respect to the time $t$. The Fourier transformation is defined by

$$
\widehat{E}(z, \Delta \omega)=\frac{1}{\sqrt{2 \pi}} \int_{-\infty}^{\infty} E(z, t) \exp (i \Delta \omega t) d t .
$$

Equation (3) represents that the temporary localized optical pulse has finite width spectrum. By setting the frequency spectrum of the optical pulse at the input of the fiber as $\widehat{E}(0, \Delta \omega)$, the frequency spectrum of the pulse, $\widehat{E}(L, \Delta \omega)$ observed after propagation of the distance $L$ in a linear fiber, the propagation constant of which is given by $\beta(\omega)$, can be calculated as

$$
\widehat{E}(L, \Delta \omega)=\exp [i \Delta \beta(\Delta \omega) L] \widehat{E}(0, \Delta \omega),
$$

where $\Delta \beta(\Delta \omega)=\beta(\omega)-\beta_{0}$. The inverse Fourier transform of Eq. (3) is given by

$$
\begin{aligned}
& E(z, t)= \frac{1}{\sqrt{2 \pi}} \int_{-\infty}^{\infty} \widehat{E}(z, \Delta \omega) \\
& \times \exp (-i \Delta \omega t) d(\Delta \omega) .
\end{aligned}
$$

Then the optical pulse observed at the output of the fiber $(z=L)$ can be calculated by substituting Eq. (4) into Eq. (5).

$$
\begin{aligned}
E(L, t)= & \frac{1}{\sqrt{2 \pi}} \int_{-\infty}^{\infty} \widehat{E}(0, \Delta \omega) \\
& \times \exp \{-i(\Delta \omega t-\Delta \beta L)\} d(\Delta \omega) .
\end{aligned}
$$

When $\Delta \beta$ is an arbitrary function of $\Delta \omega$, analytical integration of Eq. (6) is difficult. By applying Taylor expansion to the propagation constant $\beta(\omega)$ which is a function of angular frequency $\omega$, around the carrier frequency $\omega_{0}$, we have

$$
\begin{aligned}
\beta(\omega)= & \beta_{0}+\left.\frac{d \beta}{d \omega}\right|_{\omega=\omega_{0}}\left(\omega-\omega_{0}\right) \\
& +\left.\frac{1}{2} \frac{d^{2} \beta}{d \omega^{2}}\right|_{\omega=\omega_{0}}\left(\omega-\omega_{0}\right)^{2} \\
& +\left.\frac{1}{6} \frac{d^{3} \beta}{d \omega^{3}}\right|_{\omega=\omega_{0}}\left(\omega-\omega_{0}\right)^{3}+\cdots .
\end{aligned}
$$

Then

$$
\begin{aligned}
\Delta \beta(\Delta \omega)= & \beta_{0}^{(1)}(\Delta \omega)+\frac{1}{2} \beta_{0}^{(2)}(\Delta \omega)^{2} \\
& +\frac{1}{6} \beta_{0}^{(3)}(\Delta \omega)^{3}+\cdots,
\end{aligned}
$$

where

$$
\beta_{0}^{(m)}=\left.\frac{d^{m} \beta}{d \omega^{m}}\right|_{\omega=\omega_{0}}, \quad(m=1,2, \cdots) .
$$

The necessary number of expansion terms in Eq. (8) to approximate $\Delta \beta$ is dependent on the pulse width at the input of the fiber. Namely, since the bandwidth is wider for narrower pulse width, higher order term needs to be considered.

The dispersion parameter $D\left[\mathrm{~s} / \mathrm{m}^{2}\right]$ and the dispersion slope $D_{\lambda}\left[\mathrm{s} / \mathrm{m}^{3}\right]$ are frequently used to characterize a fiber. We here show the relation between $D, D_{\lambda}$ and $\beta_{0}^{(2)}\left[\mathrm{s}^{2} / \mathrm{m}\right]$, $\beta_{0}^{(3)}\left[\mathrm{s}^{3} / \mathrm{m}\right]$. $D$ is defined by the differentiation of $\beta^{(1)}$ with respect to the wavelength $\lambda$.

$$
\begin{aligned}
D & \left.\equiv \frac{d \beta^{(1)}}{d \lambda}\right|_{\omega=\omega_{0}}=\left.\frac{d}{d \lambda}\left(\frac{d \beta}{d \omega}\right)\right|_{\omega=\omega_{0}} \\
& =\left.\frac{d \omega}{d \lambda} \frac{d}{d \omega}\left(\frac{d \beta}{d \omega}\right)\right|_{\omega=\omega_{0}}=\frac{d \omega}{d \lambda} \beta_{0}^{(2)} .
\end{aligned}
$$

The relation between the angular frequency $\omega$ and the wavelength $\lambda$ is

$$
\omega=\frac{2 \pi c}{\lambda},
$$

where $c$ represents the light speed in vacuum. Then the following equation can be derived by differentiating the above equation with respect to $\lambda$ as

$$
\frac{d \omega}{d \lambda}=-\frac{2 \pi c}{\lambda^{2}} .
$$

By substituting Eq. (12) into Eq. (10), we obtain

$$
D=-\frac{2 \pi c}{\lambda^{2}} \beta_{0}^{(2)} .
$$

$D_{\lambda}$ is defined by the differentiation of $D$ with respect to $\lambda$ as

$$
\begin{aligned}
D_{\lambda} & \left.\equiv \frac{d D}{d \lambda}\right|_{\omega=\omega_{0}}=-\left.2 \pi c \frac{d}{d \lambda}\left(\frac{\beta^{(2)}}{\lambda^{2}}\right)\right|_{\omega=\omega_{0}} \\
& =-2 \pi c\left(-2 \frac{\beta_{0}^{(2)}}{\lambda^{3}}+\left.\frac{1}{\lambda^{2}} \frac{d \beta^{(2)}}{d \lambda}\right|_{\omega=\omega_{0}}\right) \\
& =\frac{4 \pi c}{\lambda^{3}} \beta_{0}^{(2)}-\left.\frac{2 \pi c}{\lambda^{2}} \frac{d \omega}{d \lambda} \frac{d}{d \omega}\left(\frac{d^{2} \beta}{d \omega^{2}}\right)\right|_{\omega=\omega_{0}} \\
& =\frac{4 \pi c}{\lambda^{3}} \beta_{0}^{(2)}+\left(\frac{2 \pi c}{\lambda^{2}}\right)^{2} \beta_{0}^{(3)} \\
& =-\frac{2 D}{\lambda}+\left(\frac{2 \pi c}{\lambda^{2}}\right)^{2} \beta_{0}^{(3)}
\end{aligned}
$$

\subsection{Nonlinear Effect in a Fiber}

Light propagating in a fiber is confined in a core which has higher refractive index than claddings and which diameter is $\mu \mathrm{m}$-order, then the intensity of the electric field is locally high and the nonlinear polarization could not be ignored. Since the fiber loss is small and the light can propagate for 
long distance with preserving its high intensity, the nonlinear optical effects are accumulated along the fiber and then obviously exhibited. In this subsection, the nonlinear optical effects in a fiber are briefly explained.

We expand the polarization vector $\boldsymbol{P}$ of the medium by the electric field $\boldsymbol{E}$ by power series as

$$
\begin{aligned}
\boldsymbol{P}= & \varepsilon_{0}\left(\chi^{(1)} \cdot \boldsymbol{E}+\chi^{(2)}: \boldsymbol{E} \boldsymbol{E}\right. \\
& \left.+\chi^{(3)}: \boldsymbol{E} \boldsymbol{E} \boldsymbol{E}+\cdots\right),
\end{aligned}
$$

where $\varepsilon_{0}$ is the permittivity in vacuum, $\chi^{(1)}$ is a linear susceptibility tensor (2nd-order tensor), $\chi^{(j)}(j \geq 2)$ is a $j$-th order nonlinear susceptibility tensor $((j+1)$-th order tensor $)$. Equation (15) shows that the polarization is proportional to the electric field and the medium can be considered as linear for weak intensity case, the nonlinear polarization can not be ignored and the nonlinear optical effects are exhibited for strong intensity case. Optical fiber is made from amorphous silica glass. Since the glass is not a crystal without noncentrosymmetry, it does not exhibit the second-order nonlinear optical effect induced by $\chi^{(2)}$ in general. The third-order nonlinear optical effect induced by $\chi^{(3)}$ is then the lowestorder effect.

Let us consider the nonlinear refractive index variation $\Delta n_{\mathrm{NL}}$ in which the index is changed with proportional to the intensity of electric field due to optical Kerr effect in one of the third-order effects.

The variation of fiber's propagation constant $\beta$, namely $\Delta \beta$, arising from the third-order effect is proportional to $\Delta n_{\mathrm{NL}}$ such like

$$
\Delta \beta=k_{0} \Delta n_{\mathrm{NL}},
$$

where $k_{0}=2 \pi / \lambda$ is the wave number in vacuum. The transmitted power through a fiber, $P[\mathrm{~W}]$ can be calculated using Eq. (2) as

$$
\begin{aligned}
& P(z, t)=\int_{S} \boldsymbol{i}_{z} \cdot(\boldsymbol{E} \times \boldsymbol{H}) d S \\
& =\frac{\beta_{0} c \varepsilon_{0}}{2 k_{0}}|E(z, t)|^{2} \int_{S}\left|\phi\left(\boldsymbol{r}_{\perp}\right)\right|^{2} d S,
\end{aligned}
$$

where $\boldsymbol{i}_{z}$ is a unit vector along the fiber axis, $\boldsymbol{H}$ is the magnetic field, and $\int_{S} d S$ represents the surface integration over the fiber's cross section. By defining

$$
E^{\prime}(z, t) \equiv \sqrt{\frac{\beta_{0} c \varepsilon_{0}}{2 k_{0}} \int_{S}\left|\phi\left(\boldsymbol{r}_{\perp}\right)\right|^{2} d S} E(z, t),
$$

based on Eq. (17), the unit of $\left|E^{\prime}(z, t)\right|^{2}$ results in [W] and then Eq. (16) can be rewritten as

$$
\Delta \beta=\gamma\left|E^{\prime}\right|^{2} E^{\prime} .
$$

Here, $\gamma[1 /(\mathrm{m} \cdot \mathrm{W})]$ is the nonlinear coefficient of optical fiber and can be represented as

$$
\gamma=k_{0} \frac{n_{2}}{A_{\text {eff }}}
$$

by using nonlinear refractive index $n_{2}\left[\mathrm{~m}^{2} / \mathrm{W}\right]$ and effective area of core $A_{\text {eff }}\left[\mathrm{m}^{2}\right] . A_{\text {eff }}$ is defined by

$$
A_{\mathrm{eff}}=\frac{\left(\int_{S}\left|\phi\left(\boldsymbol{r}_{\perp}\right)\right|^{2} d S\right)^{2}}{\int_{S}\left|\phi\left(\boldsymbol{r}_{\perp}\right)\right|^{4} d S} .
$$

2.3 The Model Equation of Optical Pulse Propagation in a Fiber

In this subsection, we derive an equation that describes the optical pulse propagation in a fiber in which both of dispersion and nonlinear effects are considered. Firstly, we focus on the dispersion effect. We define the Fourier transform of the complex amplitude of the envelope of the electric field $E(z, t)$ by

$$
\begin{aligned}
& \widetilde{E}(\Delta \beta, \Delta \omega) \equiv \frac{1}{2 \pi} \int_{-\infty}^{\infty} \int_{-\infty}^{\infty} E(z, t) \\
& \times \exp \{i(\Delta \omega t-\Delta \beta z)\} d t d z,
\end{aligned}
$$

then we have

$$
\begin{aligned}
& \frac{1}{2 \pi} \int_{-\infty}^{\infty} \int_{-\infty}^{\infty} \frac{\partial^{m} E(z, t)}{\partial t^{m}} \\
& \times \exp \{i(\Delta \omega t-\Delta \beta z)\} d t d z=(-i \Delta \omega)^{m} \widetilde{E}, \\
& \frac{1}{2 \pi} \int_{-\infty}^{\infty} \int_{-\infty}^{\infty} \frac{\partial^{m} E(z, t)}{\partial z^{m}} \\
& \times \exp \{i(\Delta \omega t-\Delta \beta z)\} d t d z=(i \Delta \beta)^{m} \widetilde{E} .
\end{aligned}
$$

By multiplying $\widetilde{E}(\Delta \beta, \Delta \omega)$ to Eq. (8), we obtain

$$
\begin{aligned}
\Delta \beta \widetilde{E}= & \beta_{0}^{(1)}(\Delta \omega) \widetilde{E}+\frac{1}{2} \beta_{0}^{(2)}(\Delta \omega)^{2} \widetilde{E} \\
& +\frac{1}{6} \beta_{0}^{(3)}(\Delta \omega)^{3} \widetilde{E}+\cdots
\end{aligned}
$$

By applying the inverse Fourier transform to Eq. (25) with considering Eqs. (23) and (24), we have

$$
\begin{aligned}
-i \frac{\partial E}{\partial z}= & i \beta_{0}^{(1)} \frac{\partial E}{\partial t}-\frac{\beta_{0}^{(2)}}{2} \frac{\partial^{2} E}{\partial t^{2}} \\
& -i \frac{\beta_{0}^{(3)}}{6} \frac{\partial^{3} E}{\partial t^{3}}+\cdots .
\end{aligned}
$$

Hereafter, higher order dispersion terms than the fourth order are omitted. Since Eq. (26) is a linear equation, the replacement from $E(z, t)$ to $E^{\prime}(z, t)$ defined by Eq. (18) does not change the form of equation. Next, considering the change of $\Delta \beta$ due to the nonlinear effect shown in Eq. (19) and adding it to the right hand side of Eq. (26), we obtain

$$
\begin{aligned}
-i \frac{\partial E^{\prime}}{\partial z}= & i \beta_{0}^{(1)} \frac{\partial E^{\prime}}{\partial t}-\frac{\beta_{0}^{(2)}}{2} \frac{\partial^{2} E^{\prime}}{\partial t^{2}} \\
& -i \frac{\beta_{0}^{(3)}}{6} \frac{\partial^{3} E^{\prime}}{\partial t^{3}}+\gamma\left|E^{\prime}\right|^{2} E^{\prime} .
\end{aligned}
$$


By adding the fiber loss term to Eq. (27), we have

$$
\begin{aligned}
& i\left(\frac{\partial E^{\prime}}{\partial z}+\beta_{0}^{(1)} \frac{\partial E^{\prime}}{\partial t}\right)-\frac{\beta_{0}^{(2)}}{2} \frac{\partial^{2} E^{\prime}}{\partial t^{2}} \\
& -i \frac{\beta_{0}^{(3)}}{6} \frac{\partial^{3} E^{\prime}}{\partial t^{3}}+\gamma\left|E^{\prime}\right|^{2} E^{\prime}=-i \alpha E^{\prime}
\end{aligned}
$$

where the loss coefficient $\alpha[1 / \mathrm{m}]$ can be represented by

$$
\alpha=\frac{\log _{e} 10}{20} \widehat{\alpha},
$$

with using $\widehat{\alpha}[\mathrm{dB} / \mathrm{m}]$. Equation (28) represents an equation which describes a lightwave propagation in an optical fiber exhibiting the dispersion up to the third-order and the nonlinear change of refractive index. Note here that we should care about the applicable range of Eq. (28) under the assumption of single mode, single polarization, and quasi-monochromatic approximation when Eq. (28) has been derived. The quasi-monochromatic approximation means that the full width at half maximum of optical spectrum, $(\Delta f)_{\mathrm{FWHM}}$, is much smaller than the carrier frequency, $f_{0}$. From Eqs. (13) and (14), $\beta_{0}^{(2)}$ and $\beta_{0}^{(3)}$ can be represented by using dispersion parameter $D$ and dispersion slope $D_{\lambda}$ as,

$$
\begin{aligned}
& \beta_{0}^{(2)}=-\frac{\lambda^{2}}{2 \pi c} D, \\
& \beta_{0}^{(3)}=\frac{\lambda^{3}}{(2 \pi c)^{2}}\left(\lambda D_{\lambda}+2 D\right) .
\end{aligned}
$$

Especially, when $\beta_{0}^{(2)}$ is not zero and the single channel optical pulse propagation in which pulse width is wider than a few picoseconds is considered, we can regard $\beta_{0}^{(3)} \sim 0$ and then the third order dispersion term can be omitted in Eq. (28).

\section{Gaussian Noise Model of Nonlinear Interference Light in Dispersion Uncompensated Optical Trans- mission Line}

A method to evaluate the influence to the transmission characteristics arising from the nonlinear optical effect in in-line dispersion uncompensated optical transmission line with using dispersion compensation by digital coherent transceiver, so-called the Gaussian noise model of nonlinear interference light is reviewed [5]-[8].

\subsection{Bit Error Rate of Digital Modulation Schemes}

In digital modulation/demodulation theory, the bit error rate (BER) can be given by a function of the (electrical) SNR [19].

$$
\mathrm{BER}=f(\mathrm{SNR}) .
$$

Definite form of a function $f$ can be determined for each modulation/demodulation scheme. The SNR is defined as a ratio of averaged signal power $P_{\mathrm{ch}}$ and noise power of amplified spontaneous emission (ASE) $P_{\mathrm{ASE}}$, and given by

$$
\mathrm{SNR}=\frac{P_{\mathrm{ch}}}{P_{\mathrm{ASE}}},
$$

where the averaged signal power $P_{\text {ch }}$ can be obtained by

$$
P_{\mathrm{ch}}=\frac{P_{R_{x}}}{R_{s}} \int_{-\infty}^{\infty}\left|H_{R_{x}}(\Delta \omega)\right|^{2} d(\Delta \omega) .
$$

Here, $P_{R_{x}}$ is the received optical power per channel and $R_{S}$ represents the symbol rate of the transmitted signal. $H_{R_{X}}(\Delta \omega)$ is the baseband transfer function of the coherent receiver. The ASE noise power $P_{\mathrm{ASE}}$ can be obtained by

$$
P_{\mathrm{ASE}}=\int_{-\infty}^{\infty} G_{\mathrm{ASE}}\left|H_{R_{X}}(\Delta \omega)\right|^{2} d(\Delta \omega),
$$

where $G_{\mathrm{ASE}}$ is a power spectral density (PSD) of a single polarization component in dual polarized ASE noise which is received at the receiver.

The relation between SNR and OSNR is given by

$$
\mathrm{OSNR}=\frac{R_{s}}{B_{n}} \mathrm{SNR},
$$

where $B_{n}$ is the OSNR bandwidth.

We assume a nonlinear interference (NLI) noise can be regarded as a Gaussian noise which is independent of ASE noise, then using the NLI noise power $P_{\mathrm{NLI}}$, the SNR including the NLI noise can be represented as

$$
\mathrm{SNR}_{\mathrm{NL}}=\frac{P_{\mathrm{ch}}}{P_{\mathrm{ASE}}+P_{\mathrm{NLI}}},
$$

where

$$
P_{\mathrm{NLI}}=\int_{-\infty}^{\infty} G_{\mathrm{NLI}}(\Delta \omega)\left|H_{R_{x}}(\Delta \omega)\right|^{2} d(\Delta \omega) .
$$

Here, $G_{\mathrm{NLI}}(\Delta \omega)$ is the PSD of the NLI noise. The relation between $\mathrm{SNR}_{\mathrm{NL}}$ and $\mathrm{OSNR}_{\mathrm{NL}}$ is then

$$
\mathrm{OSNR}_{\mathrm{NL}}=\frac{R_{s}}{B_{n}} \mathrm{SNR}_{\mathrm{NL}} \text {. }
$$

3.2 Gaussian Noise Approximation of Transmitted Optical Signal

\subsubsection{Complex Periodic White Gaussian Noise Process}

A transmitted optical signal is modelled by a complex periodic white Gaussian noise (PWGN) process with the period of $T_{0}$. It is expressed with Karhunen-Loeve expansion as

$$
E_{\mathrm{PWGN}}(t)=\frac{1}{\sqrt{T_{0}}} \sum_{n=-\infty}^{\infty} \xi_{n} \exp \left(-i n \Omega_{0} t\right),
$$

where $\Omega_{0}=2 \pi / T_{0} . \xi_{n}$ is a random variable with the mean value of 0 and the dispersion of $\sigma_{a}^{2}=1$. By applying Fourier transform to Eq. (40), we have 


$$
\begin{aligned}
& \widehat{E}_{\mathrm{PWGN}}(\Delta \omega) \\
& =\frac{1}{\sqrt{2 \pi}} \int_{-\infty}^{\infty} E_{\mathrm{PWGN}}(t) \exp (i \Delta \omega t) d t \\
& =\frac{\sqrt{\Omega_{0}}}{2 \pi} \sum_{n=-\infty}^{\infty} \xi_{n} \int_{-\infty}^{\infty} \exp \left\{i\left(\Delta \omega-n \Omega_{0}\right) t\right\} d t \\
& =\sqrt{\Omega_{0}} \sum_{n=-\infty}^{\infty} \xi_{n} \delta\left(\Delta \omega-n \Omega_{0}\right),
\end{aligned}
$$

where $\delta(\omega)$ is Dirac's delta function. Then the PSD for the PWGN is given by

$$
\begin{aligned}
& \widehat{G}_{\mathrm{PWGN}}(\Delta \omega)=\left\langle\left|\widehat{E}_{\mathrm{PWGN}}(\Delta \omega)\right|^{2}\right\rangle \\
& =\Omega_{0} \sum_{m=-\infty}^{\infty} \sum_{n=-\infty}^{\infty}\left\langle\xi_{m} \xi_{n}\right\rangle \delta\left(\Delta \omega-m \Omega_{0}\right) \delta\left(\Delta \omega-n \Omega_{0}\right) \\
& =\Omega_{0} \sum_{m=-\infty}^{\infty} \sum_{n=-\infty}^{\infty} \sigma_{a}^{2} \delta_{n m} \delta\left(\Delta \omega-m \Omega_{0}\right) \delta\left(\Delta \omega-n \Omega_{0}\right) \\
& =\Omega_{0} \sum_{n=-\infty}^{\infty} \delta\left(\Delta \omega-n \Omega_{0}\right)
\end{aligned}
$$

where $\langle *\rangle$ represents the average and $\delta_{n m}$ is Kronecker delta.

\subsubsection{Gaussian Noise Approximation of Transmitted Opti- cal Signal}

We show how to apply the Gaussian noise approximation to a transmitted optical signal $E_{a}(t) . E_{a}(t)$ is assumed as a periodic function with the period of $T_{0}$ as

$$
E_{a}(t)=q(t) \quad \text { for } 0 \leq t<T_{0} .
$$

$M$ pulses in which each temporal waveform is represented by $s(t)$ are allocated with the symbol period of $T_{s}$ in $0 \leq t<T_{0}$ and their amplitudes and/or phases are modulated by information data. $q(t)$ can be then represented as

$$
q(t)=\sum_{m=0}^{M-1} a_{m} s\left(t-m T_{s}\right)
$$

Here, $T_{0}=M T_{s}$ and $a_{m}$ is a complex number which represents the modulated amplitude and/or phase.

Next, let us calculate the Fourier transform of $E_{a}(t)$, i.e., $\widehat{E}_{a}(\Delta \omega)$. Since $E_{a}(t)$ is a periodic function with the period of $T_{0}$, it can be represented with the Fourier series expansion.

$$
E_{a}(t)=\sum_{n=-\infty}^{\infty} q_{n} \exp \left(-i n \Omega_{0} t\right)
$$

where

$$
q_{n}=\frac{1}{T_{0}} \int_{0}^{T_{0}} q(t) \exp \left(i n \Omega_{0} t\right) d t .
$$

By applying the Fourier transform to Eq. (45), we have

$$
\begin{aligned}
& \widehat{E}_{a}(\Delta \omega)=\frac{1}{\sqrt{2 \pi}} \int_{-\infty}^{\infty} E_{a}(t) \exp (i \Delta \omega t) d t \\
& =\frac{1}{\sqrt{2 \pi}} \sum_{n=-\infty}^{\infty} q_{n} \int_{-\infty}^{\infty} \exp \left\{i\left(\Delta \omega-n \Omega_{0}\right) t\right\} d t \\
& =\sqrt{2 \pi} \sum_{n=-\infty}^{\infty} q_{n} \delta\left(\Delta \omega-n \Omega_{0}\right) .
\end{aligned}
$$

By substituting Eq. (44) into Eq. (46), we obtain

$$
\begin{aligned}
q_{n}= & \frac{1}{T_{0}} \sum_{m=0}^{M-1} a_{m} \int_{0}^{T_{0}} s\left(t-m T_{s}\right) \exp \left(i n \Omega_{0} t\right) d t \\
= & \frac{1}{T_{0}} \sum_{m=0}^{M-1} a_{m} \exp \left(i n \Omega_{0} m T_{s}\right) \\
& \times \int_{-m T_{s}}^{T_{0}-m T_{s}} s(\tau) \exp \left(i n \Omega_{0} \tau\right) d \tau
\end{aligned}
$$

Here, we assume

$$
\begin{aligned}
\widehat{s}\left(n \Omega_{0}\right) & =\frac{1}{\sqrt{2 \pi}} \int_{-m T_{s}}^{T_{0}-m T_{s}} s(\tau) \exp \left(i n \Omega_{0} \tau\right) d \tau \\
& \simeq \frac{1}{\sqrt{2 \pi}} \int_{-\infty}^{\infty} s(\tau) \exp \left(i n \Omega_{0} \tau\right) d \tau,
\end{aligned}
$$

and use $\Omega_{0}=2 \pi / T_{0}$ and $T_{s}=T_{0} / M$, Eq. (48) can be represented as

$$
q_{n}=\frac{\sqrt{2 \pi}}{T_{0}} \widehat{s}\left(n \Omega_{0}\right) \sum_{m=0}^{M-1} a_{m} \exp \left(i 2 \pi n \frac{m}{M}\right) .
$$

By substituting Eq. (50) into Eq. (47), we have

$$
\begin{aligned}
\widehat{E}_{a}(\Delta \omega)= & \Omega_{0} \sum_{n=-\infty}^{\infty} \widehat{s}\left(n \Omega_{0}\right) \delta\left(\Delta \omega-n \Omega_{0}\right) \\
& \times \sum_{m=0}^{M-1} a_{m} \exp \left(i 2 \pi n \frac{m}{M}\right) .
\end{aligned}
$$

Considering the property of Dirac's delta function, $\widehat{s}\left(n \Omega_{0}\right) \delta\left(\Delta \omega-n \Omega_{0}\right)$ can be replaced by $\widehat{s}(\Delta \omega)$ $\delta\left(\Delta \omega-n \Omega_{0}\right)$ in Eq. (51). Therefore,

$$
\begin{aligned}
\widehat{E}_{a}(\Delta \omega)= & \Omega_{0} \widehat{s}(\Delta \omega) \sum_{n=-\infty}^{\infty} \delta\left(\Delta \omega-n \Omega_{0}\right) \\
& \times \sum_{m=0}^{M-1} a_{m} \exp \left(i 2 \pi n \frac{m}{M}\right) .
\end{aligned}
$$

Furthermore, we assume

$$
\widehat{s}(\Delta \omega)=|\widehat{s}(\Delta \omega)| \exp \left\{i \varphi_{s}(\Delta \omega)\right\},
$$

and rewrite Eq. (52) as

$$
\begin{aligned}
\widehat{E}_{a}(\Delta \omega)= & \sqrt{\Omega_{0}}|\widehat{s}(\Delta \omega)| \\
& \times \sum_{n=-\infty}^{\infty} \zeta_{n} \delta\left(\Delta \omega-n \Omega_{0}\right) .
\end{aligned}
$$

Here, $\zeta_{n}$ is a random variable defined by 


$$
\begin{aligned}
\zeta_{n}= & \sqrt{\Omega_{0}} \sum_{m=0}^{M-1} a_{m} \\
& \times \exp \left[i\left\{2 \pi n \frac{m}{M}+\varphi_{s}\left(n \Omega_{0}\right)\right\}\right] .
\end{aligned}
$$

By calculating the average value of $\zeta_{n} \zeta_{\ell}^{*}$, we have

$$
\begin{aligned}
\left\langle\zeta_{n} \zeta_{\ell}^{*}\right\rangle= & \Omega_{0}\left\langle\sum_{m_{1}=0}^{M-1} a_{m_{1}} \exp \left[i\left\{2 \pi n \frac{m_{1}}{M}+\varphi_{s}\left(n \Omega_{0}\right)\right\}\right]\right. \\
& \left.\times \sum_{m_{2}=0}^{M-1} a_{m_{2}}^{*} \exp \left[-i\left\{2 \pi \ell \frac{m_{2}}{M}+\varphi_{s}\left(\ell \Omega_{0}\right)\right\}\right]\right\rangle \\
= & \Omega_{0} \exp \left[i\left\{\varphi_{s}\left(n \Omega_{0}\right)-\varphi_{s}\left(\ell \Omega_{0}\right)\right\}\right] \\
& \times \sum_{m_{1}=0}^{M-1} \sum_{m_{2}=0}^{M-1}\left\langle a_{m_{1}} a_{m_{2}}^{*}\right\rangle \exp \left(i 2 \pi \frac{n m_{1}-\ell m_{2}}{M}\right) \\
= & \Omega_{0} \sigma_{a}^{2} \exp \left[i\left\{\varphi_{s}\left(n \Omega_{0}\right)-\varphi_{s}\left(\ell \Omega_{0}\right)\right\}\right] \\
& \times \sum_{m_{1}=0}^{M-1} \sum_{m_{2}=0}^{M-1} \delta_{m_{1} m_{2}} \exp \left(i 2 \pi \frac{n m_{1}-\ell m_{2}}{M}\right) \\
= & \Omega_{0} \sigma_{a}^{2} \exp \left[i\left\{\varphi_{s}\left(n \Omega_{0}\right)-\varphi_{s}\left(\ell \Omega_{0}\right)\right\}\right] \\
& \times \sum_{m=0}^{M-1} \exp \left\{i 2 \pi(n-\ell) \frac{m}{M}\right\}
\end{aligned}
$$

where $\sigma_{a}^{2}$ is a variance of the Gaussian variable of $\zeta_{n}$, and

$$
\begin{aligned}
& \sum_{m=0}^{M-1} \exp \left\{i 2 \pi(n-\ell) \frac{m}{M}\right\} \\
& = \begin{cases}0 & \text { if } n \neq \ell \text { and }(n-\ell) \neq k M, \\
M & \text { if }(n-\ell)=p M .\end{cases}
\end{aligned}
$$

\subsubsection{Model of Transmitted Optical Signal}

Based on the discussions in Sects. 3.2.1 and 3.2.2, using Eq. (41), the transmitted optical signal approximated by the Gaussian noise model is represented by

$$
\begin{aligned}
& \widehat{E}_{\mathrm{GN}}(\Delta \omega)=\sqrt{G_{T_{x}}(\Delta \omega)} \widehat{E}_{\mathrm{PWGN}}(\Delta \omega) \\
& =\sqrt{G_{T_{x}}(\Delta \omega)} \sqrt{\Omega_{0}} \sum_{n=-\infty}^{\infty} \xi_{n} \delta\left(\Delta \omega-n \Omega_{0}\right) \\
& =\sqrt{\Omega_{0}} \sum_{n=-\infty}^{\infty} \xi_{n} \sqrt{G_{T_{x}}\left(n \Omega_{0}\right)} \delta\left(\Delta \omega-n \Omega_{0}\right) \\
& =\sum_{n=-\infty}^{\infty} \widehat{E}_{n}(0) \delta\left(\Delta \omega-n \Omega_{0}\right),
\end{aligned}
$$

where

$$
\widehat{E}_{n}(0)=\xi_{n} \sqrt{\Omega_{0} G_{T_{x}}\left(n \Omega_{0}\right)} .
$$

Here, $G_{T_{x}}(\Delta \omega)$ represents the envelope of modulated spectrum of each channel in the transmitter. Then the PSD of the transmitted signal is given by

$$
\begin{aligned}
& \widehat{G}_{\mathrm{GN}}(\Delta \omega)=\left\langle\left|\widehat{E}_{\mathrm{GN}}(\Delta \omega)\right|^{2}\right\rangle \\
& =\Omega_{0} G_{T_{x}}(\Delta \omega) \sum_{m=-\infty}^{\infty} \sum_{n=-\infty}^{\infty}\left\langle\xi_{m} \xi_{n}^{*}\right\rangle \\
& \times \delta\left(\Delta \omega-m \Omega_{0}\right) \delta\left(\Delta \omega-n \Omega_{0}\right) \\
& =\Omega_{0} G_{T_{x}}(\Delta \omega) \sum_{m=-\infty}^{\infty} \sum_{n=-\infty}^{\infty} \delta_{m n} \sigma_{a}^{2} \\
& \times \delta\left(\Delta \omega-m \Omega_{0}\right) \delta\left(\Delta \omega-n \Omega_{0}\right) \\
& =\Omega_{0} G_{T_{x}}(\Delta \omega) \sum_{n=-\infty}^{\infty} \delta\left(\Delta \omega-n \Omega_{0}\right) .
\end{aligned}
$$

Next, the inverse Fourier transform of Eq. (58) leads

$$
\begin{aligned}
E_{\mathrm{GN}}(t)= & \frac{1}{\sqrt{2 \pi}} \int_{-\infty}^{\infty} \widehat{E}_{\mathrm{GN}}(\Delta \omega) \exp (-i \Delta \omega t) d(\Delta \omega) \\
= & \sum_{n=-\infty}^{\infty} \frac{\widehat{E}_{n}(0)}{\sqrt{2 \pi}} \int_{-\infty}^{\infty} \delta\left(\Delta \omega-n \Omega_{0}\right) \\
& \times \exp (-i \Delta \omega t) d(\Delta \omega) \\
= & \sum_{n=-\infty}^{\infty} \frac{\widehat{E}_{n}(0)}{\sqrt{2 \pi}} \exp \left(-i n \Omega_{0} t\right) .
\end{aligned}
$$

Using the input waveform to the transmission line shown in Eq. (61), let us calculate the electric field after propagating through the transmission line in the next subsections.

3.3 Nonlinear Interference Noise Generated by Four Wave Mixing

\subsubsection{Non-degenerate Four Wave Mixing Model}

For the simplicity, by picking up 4 different frequency continuous lights in the discrete spectrum shown in Eq. (61) and considering those lights propagation in a fiber, we set $E(z, t)$ as

$$
E(z, t)=\sum_{k=1}^{4} \frac{\widehat{E}_{k}(z)}{\sqrt{2 \pi}} \exp \left(-i \Delta \omega_{k} t\right)
$$

where $\Delta \omega_{k}(k=1,2,3,4)$ is the difference between the angular frequency $\omega_{k}$ and the reference angular frequency $\omega_{0}$ in Eq. (2), i.e., $\Delta \omega_{k}=\omega_{k}-\omega_{0}$. The frequency allocation is assumed to satisfy

$$
\omega_{3}+\omega_{4}=\omega_{1}+\omega_{2} .
$$

Under this condition, $\Delta \omega_{k}$ satisfies

$$
\Delta \omega_{3}+\Delta \omega_{4}=\Delta \omega_{1}+\Delta \omega_{2},
$$

as shown in Fig. 1. Hereafter, we consider the case in which $\Delta \omega_{2}+\Delta \omega_{3} \neq 2 \Delta \omega_{1}$ is satisfied. By substituting Eq. (64) into Eq. (28), Eq. (28) can be separated to four equations as

$$
\begin{aligned}
& i \frac{d \widehat{E}_{1}}{d z}+\Delta \beta\left(\Delta \omega_{1}\right) \widehat{E}_{1} \\
& +\frac{\gamma}{2 \pi}\left\{\left(\left|\widehat{E}_{1}\right|^{2}+2 \sum_{i=2,3,4}\left|\widehat{E}_{i}\right|^{2}\right) \widehat{E}_{1}+2 \widehat{E}_{2}^{*} \widehat{E}_{3} \widehat{E}_{4}\right\} \\
& =-i \alpha \widehat{E}_{1},
\end{aligned}
$$




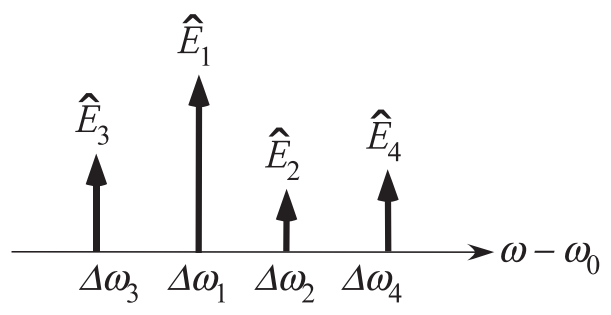

Fig. 1 Frequency allocation of four continuous waves in which nondegenerate four wave mixing occurs.

$$
\begin{aligned}
& i \frac{d \widehat{E}_{2}}{d z}+\Delta \beta\left(\Delta \omega_{2}\right) \widehat{E}_{2} \\
& +\frac{\gamma}{2 \pi}\left\{\left(\left|\widehat{E}_{2}\right|^{2}+2 \sum_{i=1,3,4}\left|\widehat{E}_{i}\right|^{2}\right) \widehat{E}_{2}+2 \widehat{E}_{1}^{*} \widehat{E}_{3} \widehat{E}_{4}\right\} \\
& =-i \alpha \widehat{E}_{2}, \\
& i \frac{d \widehat{E}_{3}}{d z}+\Delta \beta\left(\Delta \omega_{3}\right) \widehat{E}_{3} \\
& +\frac{\gamma}{2 \pi}\left\{\left(\left|\widehat{E}_{3}\right|^{2}+2 \sum_{i=1,2,4}\left|\widehat{E}_{i}\right|^{2}\right) \widehat{E}_{3}+2 \widehat{E}_{1} \widehat{E}_{2} \widehat{E}_{4}^{*}\right\} \\
& =-i \alpha \widehat{E}_{3}, \\
& i \frac{d \widehat{E}_{4}}{d z}+\Delta \beta\left(\Delta \omega_{4}\right) \widehat{E}_{4} \\
& +\frac{\gamma}{2 \pi}\left\{\left(\left|\widehat{E}_{4}\right|^{2}+2 \sum_{i=1,2,3}\left|\widehat{E}_{i}\right|^{2}\right) \widehat{E}_{4}+2 \widehat{E}_{1} \widehat{E}_{2} \widehat{E}_{3}^{*}\right\} \\
& =-i \alpha \widehat{E}_{4},
\end{aligned}
$$

where

$$
\begin{aligned}
\Delta \beta(\Delta \omega) & =\beta_{0}^{(1)}(\Delta \omega)+\frac{\beta_{0}^{(2)}}{2}(\Delta \omega)^{2} \\
& +\frac{\beta_{0}^{(3)}}{6}(\Delta \omega)^{3} .
\end{aligned}
$$

In the nonlinear terms in Eqs. (65)-(68), we focus on the four wave mixing (FWM) terms. The term of $\gamma \widehat{E}_{1} \widehat{E}_{2} \widehat{E}_{3}^{*} / \pi$ in Eq. (68) represents a continuous light generation at $\omega_{4}\left(=\omega_{1}+\omega_{2}-\omega_{3}\right)$ by the nonlinear interactions among three continuous lights at the angular frequency of $\omega_{1}, \omega_{2}$, and $\omega_{3}$. This is called the non-degenerate FWM.

Hereafter, we consider a case where the idler light $\widehat{E}_{4}$ is generated by the non-degenerate FWM when three lights, $\widehat{E}_{1}$, $\widehat{E}_{2}$, and $\widehat{E}_{3}$, are simultaneously launched into a fiber. Omitting all nonlinear terms in Eqs. (65)-(67) and the nonlinear terms representing the self phase modulation (SPM) and the cross phase modulation (XPM) in Eq. (68), Eqs. (65)-(68) can be rewritten as

$$
\begin{gathered}
\frac{d \widehat{E}_{j}}{d z}+\left\{\alpha-i \Delta \beta\left(\Delta \omega_{j}\right)\right\} \widehat{E}_{j}=0 \text { for } j=1,2,3 \\
\frac{d \widehat{E}_{4}}{d z}+\left\{\alpha-i \Delta \beta\left(\Delta \omega_{4}\right)\right\} \widehat{E}_{4}-i \frac{\gamma}{\pi} \widehat{E}_{1} \widehat{E}_{2} \widehat{E}_{3}^{*}=0 .
\end{gathered}
$$

The solution of Eq. (70) is

$$
\widehat{E}_{j}(z)=\widehat{E}_{j}(0) \exp \left[\left\{-\alpha+i \Delta \beta\left(\Delta \omega_{j}\right)\right\} z\right] .
$$

We assume that the solution of Eq. (71) has the following form,

$$
\widehat{E}_{4}(z)=\widetilde{E}_{4}(z) \exp \left[\left\{-\alpha+i \Delta \beta\left(\Delta \omega_{4}\right)\right\} z\right],
$$

where $\widetilde{E}_{4}(z)$ is a complex function of $z$. By substituting Eqs. (72) and (73) into Eq. (71), we obtain

$$
\begin{aligned}
\frac{d \widetilde{E}_{4}}{d z} & =i \frac{\gamma}{\pi} \widehat{E}_{1}(0) \widehat{E}_{2}(0) \widehat{E}_{3}^{*}(0) \\
& \times \exp [(-2 \alpha+i \Delta B) z],
\end{aligned}
$$

where $\Delta B$ is the phase mismatching parameter. By using Eqs. (69) and (64), $\Delta B$ can be represented by

$$
\begin{aligned}
\Delta B= & \Delta \beta_{1}+\Delta \beta_{2}-\Delta \beta_{3}-\Delta \beta_{4} \\
= & \beta_{0}^{(1)}\left(\Delta \omega_{1}+\Delta \omega_{2}-\Delta \omega_{3}-\Delta \omega_{4}\right) \\
& +\frac{\beta_{0}^{(2)}}{2}\left\{\left(\Delta \omega_{1}\right)^{2}+\left(\Delta \omega_{2}\right)^{2}-\left(\Delta \omega_{3}\right)^{2}-\left(\Delta \omega_{4}\right)^{2}\right\} \\
& +\frac{\beta_{0}^{(3)}}{6}\left\{\left(\Delta \omega_{1}\right)^{3}+\left(\Delta \omega_{2}\right)^{3}-\left(\Delta \omega_{3}\right)^{3}-\left(\Delta \omega_{4}\right)^{3}\right\} \\
= & -\beta_{0}^{(2)}\left(\Delta \omega_{4}-\Delta \omega_{1}\right)\left(\Delta \omega_{4}-\Delta \omega_{2}\right) \\
& -\frac{\beta_{0}^{(3)}}{2}\left(\Delta \omega_{1}+\Delta \omega_{2}\right)\left(\Delta \omega_{4}-\Delta \omega_{1}\right)\left(\Delta \omega_{4}-\Delta \omega_{2}\right) \\
= & -\left(\Delta \omega_{4}-\Delta \omega_{1}\right)\left(\Delta \omega_{4}-\Delta \omega_{2}\right) \\
& \times\left\{\beta_{0}^{(2)}+\beta_{0}^{(3)} \frac{\Delta \omega_{1}+\Delta \omega_{2}}{2}\right\} .
\end{aligned}
$$

By setting $\widetilde{E}_{4}(0)=0$, Eq. (74) can be integrated as

$$
\begin{aligned}
& \widetilde{E}_{4}(z)=i \frac{\gamma}{\pi} \widehat{E}_{1}(0) \widehat{E}_{2}(0) \widehat{E}_{3}^{*}(0) \\
& \times \frac{1-\exp [(-2 \alpha+i \Delta B) z]}{2 \alpha-i \Delta B} .
\end{aligned}
$$

By substituting Eq. (76) into Eq. (73), $\widehat{E}_{4}(z)$ can be rewritten as

$$
\begin{aligned}
& \widehat{E}_{4}(z)=i \frac{\gamma}{\pi} \widehat{E}_{1}(0) \widehat{E}_{2}(0) \widehat{E}_{3}^{*}(0) \\
& \times \frac{1-\exp [(-2 \alpha+i \Delta B) z]}{2 \alpha-i \Delta B} \exp \left[\left\{-\alpha+i \Delta \beta\left(\Delta \omega_{4}\right)\right\} z\right] .
\end{aligned}
$$

\subsubsection{FWM Light Generated in Periodically Amplified Transmission System}

Let us consider the FWM light generated in periodically amplified optical transmission system in which optical amplifiers are disposed with the spacing of $z_{a}$ [20], [21]. By considering $N$ amplifiers, the total system length is $N z_{a}$. The transmission fiber is assumed to be homogeneous in which 
the group velocity dispersion $\beta_{0}^{(2)}$, the third order dispersion $\beta_{0}^{(3)}$, the nonlinear coefficient $\gamma$, and the loss coefficient $\alpha$ are constant. Furthermore, optical amplifiers are placed just before the output end of each section and their power gain $G$ is set to $G=\exp \left(2 \alpha z_{a}\right)$ with which the loss of one section with the length $z_{a}$ can be compensated.

FWM light generated in each section independently propagates in the remaining transmission line linearly and reaches to the output. At the output, the total FWM light is obtained by coherently summing up the FWM lights generated in each section.

Firstly, the electric field of FWM light $\widehat{E}_{4}^{(k)}\left(k z_{a}\right)$ at the output of the $k$-th section which is generated in the $k$-th section can be calculated by using Eq. (77) as

$$
\begin{aligned}
& \widehat{E}_{4}^{(k)}\left(k z_{a}\right)=i \frac{\gamma}{\pi} \widehat{E}_{1}\left[(k-1) z_{a}\right] \widehat{E}_{2}\left[(k-1) z_{a}\right] \\
& \times \widehat{E}_{3}^{*}\left[(k-1) z_{a}\right] \frac{1-\exp \left[(-2 \alpha+i \Delta B) z_{a}\right]}{2 \alpha-i \Delta B} \\
& \times \exp \left[\left\{-\alpha+i \Delta \beta\left(\Delta \omega_{4}\right)\right\} z_{a}\right] \sqrt{G} .
\end{aligned}
$$

The electric field $\widehat{E}_{j}\left[(k-1) z_{a}\right](j=1,2,3)$ at the input of the $k$-th section can be calculated by using Eq. (72) as

$$
\begin{aligned}
\widehat{E}_{j}\left[(k-1) z_{a}\right]= & \widehat{E}_{j}(0) \\
& \times \exp \left[i \Delta \beta\left(\Delta \omega_{j}\right)(k-1) z_{a}\right] .
\end{aligned}
$$

By substituting Eq. (79) into Eq. (78), we have

$$
\begin{aligned}
& \widehat{E}_{4}^{(k)}\left(k z_{a}\right)=i \frac{\gamma}{\pi} \widehat{E}_{1}(0) \widehat{E}_{2}(0) \widehat{E}_{3}^{*}(0) \\
& \times \exp \left[i\left\{\Delta \beta\left(\Delta \omega_{1}\right)+\Delta \beta\left(\Delta \omega_{2}\right)-\Delta \beta\left(\Delta \omega_{3}\right)\right\}(k-1) z_{a}\right] \\
& \times \exp \left[i \Delta \beta\left(\Delta \omega_{4}\right) z_{a}\right] \frac{1-\exp \left[(-2 \alpha+i \Delta B) z_{a}\right]}{2 \alpha-i \Delta B} \\
& =i \frac{\gamma}{\pi} \widehat{E}_{1}(0) \widehat{E}_{2}(0) \widehat{E}_{3}^{*}(0) \\
& \times \exp \left[i\left\{\Delta B+\Delta \beta\left(\Delta \omega_{4}\right)\right\}(k-1) z_{a}\right] \\
& \times \exp \left[i \Delta \beta\left(\Delta \omega_{4}\right) z_{a}\right] \frac{1-\exp \left[(-2 \alpha+i \Delta B) z_{a}\right]}{2 \alpha-i \Delta B} \\
& =i \frac{\gamma}{\pi} \widehat{E}_{1}(0) \widehat{E}_{2}(0) \widehat{E}_{3}^{*}(0) \exp \left[i \Delta B(k-1) z_{a}\right] \\
& \times \exp \left[i \Delta \beta\left(\Delta \omega_{4}\right) k z_{a}\right] \frac{1-\exp \left[(-2 \alpha+i \Delta B) z_{a}\right]}{2 \alpha-i \Delta B} .
\end{aligned}
$$

The FWM light $\widehat{E}_{4}^{(k)}\left(k z_{a}\right)$ generated in the $k$-th section is assumed to propagate in the remaining transmission line linearly and reach the output end of the transmission line. Then the FWM light $\widehat{E}_{4}^{(k)}\left(N z_{a}\right)$ at the output $z=N z_{a}$ can be calculated by using Eq. (72) as

$$
\begin{aligned}
& \widehat{E}_{4}^{(k)}\left(N z_{a}\right)=\widehat{E}_{4}^{(k)}\left(k z_{a}\right) \exp \left[i \Delta \beta\left(\Delta \omega_{4}\right)(N-k) z_{a}\right] \\
& =i \frac{\gamma}{\pi} \widehat{E}_{1}(0) \widehat{E}_{2}(0) \widehat{E}_{3}^{*}(0) \exp \left[i \Delta B(k-1) z_{a}\right] \\
& \times \exp \left[i \Delta \beta\left(\Delta \omega_{4}\right) N z_{a}\right] \frac{1-\exp \left[(-2 \alpha+i \Delta B) z_{a}\right]}{2 \alpha-i \Delta B} .
\end{aligned}
$$

By summing up Eq. (81) for all $k$, the total FWM light $\widehat{E}_{4}^{\text {(total) }}\left(N z_{a}\right)$ at the output $z=N z_{a}$ can be obtained as

$$
\begin{aligned}
& \widehat{E}_{4}^{\text {(toal) }}\left(N z_{a}\right)=\sum_{k=1}^{N} \widehat{E}_{4}^{(k)}\left(N z_{a}\right) \\
& =i \frac{\gamma}{\pi} \widehat{E}_{1}(0) \widehat{E}_{2}(0) \widehat{E}_{3}^{*}(0) \frac{1-\exp \left[(-2 \alpha+i \Delta B) z_{a}\right]}{2 \alpha-i \Delta B} \\
& \times \exp \left[i \Delta \beta\left(\Delta \omega_{4}\right) N z_{a}\right] \sum_{k=1}^{N} \exp \left[i \Delta B(k-1) z_{a}\right] \\
& =i \frac{\gamma}{\pi} \widehat{E}_{1}(0) \widehat{E}_{2}(0) \widehat{E}_{3}^{*}(0) \frac{1-\exp \left[(-2 \alpha+i \Delta B) z_{a}\right]}{2 \alpha-i \Delta B} \\
& \times \exp \left[i \Delta \beta\left(\Delta \omega_{4}\right) N z_{a}\right] \frac{1-\exp \left(i \Delta B N z_{a}\right)}{1-\exp \left(i \Delta B z_{a}\right)} \\
& =i \frac{\gamma}{\pi} \widehat{E}_{1}(0) \widehat{E}_{2}(0) \widehat{E}_{3}^{*}(0) \frac{1-\exp \left[(-2 \alpha+i \Delta B) z_{a}\right]}{2 \alpha-i \Delta B} \\
& \times \exp \left[i\left\{\Delta \beta\left(\Delta \omega_{4}\right) N+\frac{\Delta B(N-1)}{2}\right\} z_{a}\right] \\
& \quad \sin \left(\frac{\Delta B N z_{a}}{2}\right) \\
& \times \frac{\sin \left(\frac{\Delta B z_{a}}{2}\right)}{}
\end{aligned}
$$

\subsection{Gaussian Noise Model}

By setting $\quad \Delta \omega_{1}=\ell \Omega_{0}, \quad \Delta \omega_{2}=m \Omega_{0}, \quad$ and $\quad \Delta \omega_{3}=$ $(\ell+m-n) \Omega_{0}, \Delta \omega_{4}=n \Omega_{0}$, and rewriting $\Delta B$ as $\Delta B_{\ell m n}$ in Eq. (75), we have

$$
\begin{aligned}
\Delta B_{\ell m n} & =(n-\ell)(n-m) \Omega_{0}^{2} \\
& \times\left\{\beta_{0}^{(2)}+\beta_{0}^{(3)} \frac{(\ell+m) \Omega_{0}}{2}\right\} .
\end{aligned}
$$

The FWM term $\gamma \widehat{E}_{1} \widehat{E}_{2} \widehat{E}_{3}^{*} / \pi$ in Eq. (68) can be represented by the sum of exchanging the order of $\widehat{E}_{1}$ and $\widehat{E}_{2}$, as $\gamma\left(\widehat{E}_{1} \widehat{E}_{2}+\widehat{E}_{2} \widehat{E}_{1}\right) \widehat{E}_{3}^{*} /(2 \pi)$. Therefore, changing the order of $\ell$ and $m$, and counting them separately, the right hand side of Eqs. (77) and (82) becomes half. By rewriting $\widehat{E}_{4}(z)$ to $\widehat{E}\left(z, n \Omega_{0}\right)$ in these equations and substituting Eq. (59) into them, we have

$$
\begin{aligned}
& \widehat{E}\left(z, n \Omega_{0}\right)=i \frac{\gamma}{2 \pi} \Omega_{0}^{3 / 2} \xi_{\ell} \xi_{m} \xi_{\ell+m-n}^{*} \\
& \times \sqrt{G_{T_{x}}\left(\ell \Omega_{0}\right) G_{T_{x}}\left(m \Omega_{0}\right) G_{T_{x}}\left[(\ell+m-n) \Omega_{0}\right]} \\
& \times \frac{1-\exp \left[\left(-2 \alpha+i \Delta B_{\ell m n}\right) z\right]}{2 \alpha-i \Delta B_{\ell m n}} \\
& \times \exp \left[\left\{-\alpha+i \Delta \beta\left(n \Omega_{0}\right)\right\} z\right], \\
& \widehat{E}^{\text {(total })}\left(N z_{a}, n \Omega_{0}\right)=i \frac{\gamma}{2 \pi} \Omega_{0}^{3 / 2} \xi_{\ell} \xi_{m} \xi_{\ell+m-n}^{*} \\
& \times \sqrt{G_{T_{x}}\left(\ell \Omega_{0}\right) G_{T_{x}}\left(m \Omega_{0}\right) G_{T_{x}}\left[(\ell+m-n) \Omega_{0}\right]} \\
& \times \frac{1-\exp \left[\left(-2 \alpha+i \Delta B_{\ell m n}\right) z_{a}\right]}{2 \alpha-i \Delta B_{\ell m n}} \frac{\sin \left(\frac{\Delta B_{\ell m n} N z_{a}}{2}\right)}{\sin \left(\frac{\Delta B_{\ell m n} z_{a}}{2}\right)}
\end{aligned}
$$




$$
\times \exp \left[i\left\{\Delta \beta\left(n \Omega_{0}\right) N+\frac{\Delta B_{\ell m n}(N-1)}{2}\right\} z_{a}\right]
$$

\subsubsection{Gaussian Noise Model for Incoherent FWM Light}

When the FWM light generated in a single span (ss) is incoherent, the FWM light generated in all sections can be obtained by summing up the power of FWM light generated in $N$ sections.

In periodically amplified optical transmission system, by representing the power gain of optical amplifier located at just before the output end of each section as $G=\exp \left(2 \alpha z_{a}\right)$, nonlinear interference light $\widehat{E}_{\mathrm{NLI}}^{(\mathrm{ss})}\left(z_{a}, \Delta \omega\right)$ generated in a single span can be expressed as

$$
\begin{aligned}
& \widehat{E}_{\mathrm{NLI}}^{(\mathrm{ss})}\left(z_{a}, \Delta \omega\right) \\
& =\sum_{n=-\infty}^{\infty} \widehat{E}_{\mathrm{NLI}}^{(\mathrm{ss})}\left(z_{a}, n \Omega_{0}\right) \sqrt{G} \delta\left(\Delta \omega-n \Omega_{0}\right) .
\end{aligned}
$$

$\widehat{E}_{\mathrm{NLI}}^{\mathrm{ss}}\left(z_{a}, n \Omega_{0}\right)$ can be calculated by using Eq. (84) as

$$
\begin{aligned}
& \widehat{E}_{\mathrm{NLI}}^{\mathrm{ss}}\left(z_{a}, n \Omega_{0}\right)=\sum_{\ell} \sum_{m} \widehat{E}\left(z_{a}, n \Omega_{0}\right) \\
& =i \frac{\gamma}{2 \pi} \Omega_{0}^{3 / 2} \exp \left[\left\{-\alpha+i \Delta \beta\left(n \Omega_{0}\right)\right\} z_{a}\right] \\
& \times \sum_{\ell} \sum_{m} \xi_{\ell} \xi_{m} \xi_{\ell+m-n}^{*} \\
& \times \sqrt{G_{T_{x}}\left(\ell \Omega_{0}\right) G_{T_{x}}\left(m \Omega_{0}\right) G_{T_{x}}\left[(\ell+m-n) \Omega_{0}\right]} \\
& \times \frac{1-\exp \left[-\left\{2 \alpha-i \Delta B_{\ell m n}\right\} z_{a}\right]}{2 \alpha-i \Delta B_{\ell m n}} .
\end{aligned}
$$

Therefore the NLI power $\widehat{P}_{\mathrm{NLI}}^{(\mathrm{ss})}\left(z_{a}, n \Omega_{0}\right)$ is represented as

$$
\begin{aligned}
& \widehat{P}_{\mathrm{NLI}}^{\mathrm{ss}}\left(z_{a}, n \Omega_{0}\right)=\left\langle\left|\widehat{E}_{\mathrm{NLI}}^{\mathrm{ss})}\left(z_{a}, n \Omega_{0}\right)\right|^{2} G\right\rangle \\
& =\left\langle\left(\frac{\gamma}{2 \pi}\right)^{2} \Omega_{0}^{3} \sum_{\ell} \sum_{m} \xi_{\ell} \xi_{m} \xi_{\ell+m-n}^{*}\right. \\
& \times \sqrt{G_{T_{x}}\left(\ell \Omega_{0}\right) G_{T_{x}}\left(m \Omega_{0}\right) G_{T_{x}}\left[(\ell+m-n) \Omega_{0}\right]} \\
& \times \frac{1-\exp \left[-\left\{2 \alpha-i \Delta B_{\ell m n}\right\} z_{a}\right]}{2 \alpha-i \Delta B_{\ell m n}} \\
& \times \sum_{\ell^{\prime}} \sum_{m^{\prime}} \xi_{\ell^{\prime}}^{*} \xi_{m^{\prime}}^{*} \xi_{\ell^{\prime}+m^{\prime}-n} \\
& \times \sqrt{G_{T_{x}}\left(\ell^{\prime} \Omega_{0}\right) G_{T_{x}}\left(m^{\prime} \Omega_{0}\right) G_{T_{x}}\left[\left(\ell^{\prime}+m^{\prime}-n\right) \Omega_{0}\right]} \\
& \left.\times \frac{1-\exp \left[-\left\{2 \alpha+i \Delta B_{\ell^{\prime} m^{\prime} n}\right\} z_{a}\right]}{2 \alpha+i \Delta B_{\ell^{\prime} m^{\prime} n}}\right\rangle \\
& =\left(\frac{\gamma}{2 \pi}\right)^{2} \Omega_{0}^{3} \sum_{\ell} \sum_{m} \sum_{\ell^{\prime}} \sum_{m^{\prime}} \\
& \left\langle\xi_{\ell} \xi_{m} \xi_{\ell+m-n}^{*} \xi_{\ell^{\prime}}^{*} \xi_{m^{\prime}}^{*} \xi_{\ell^{\prime}+m^{\prime}-n}\right\rangle \\
& \times \sqrt{G_{T_{x}}\left(\ell \Omega_{0}\right) G_{T_{x}}\left(m \Omega_{0}\right) G_{T_{x}}\left[(\ell+m-n) \Omega_{0}\right]}
\end{aligned}
$$

$$
\begin{aligned}
& \times \sqrt{G_{T_{x}}\left(\ell^{\prime} \Omega_{0}\right) G_{T_{x}}\left(m^{\prime} \Omega_{0}\right) G_{T_{x}}\left[\left(\ell^{\prime}+m^{\prime}-n\right) \Omega_{0}\right]} \\
& \times \frac{1-\exp \left[-\left\{2 \alpha-i \Delta B_{\ell m n}\right\} z_{a}\right]}{2 \alpha-i \Delta B_{\ell m n}} \\
& \times \frac{1-\exp \left[-\left\{2 \alpha+i \Delta B_{\ell^{\prime} m^{\prime} n}\right\} z_{a}\right]}{2 \alpha+i \Delta B_{\ell^{\prime} m^{\prime} n}} \\
& =\left(\frac{\gamma}{2 \pi}\right)^{2} \Omega_{0}^{3} \sum_{\ell} \sum_{\ell^{\prime}}\left\langle\xi_{\ell} \xi_{\ell^{\prime}}^{*}\right\rangle \\
& \left.\times \sum_{m} \sum_{m^{\prime}}\left\langle\xi_{m} \xi_{m^{\prime}}^{*}\right\rangle \xi_{\ell+m-n}^{*} \xi_{\ell^{\prime}+m^{\prime}-n}\right\rangle \\
& \times \sqrt{G_{T_{x}}\left(\ell \Omega_{0}\right) G_{T_{x}}\left(m \Omega_{0}\right) G_{T_{x}}\left[(\ell+m-n) \Omega_{0}\right]} \\
& \times \sqrt{G_{T_{x}}\left(\ell^{\prime} \Omega_{0}\right) G_{T_{x}}\left(m^{\prime} \Omega_{0}\right) G_{T_{x}}\left[\left(\ell^{\prime}+m^{\prime}-n\right) \Omega_{0}\right]} \\
& \times \frac{1-\exp \left[-\left\{2 \alpha-i \Delta B_{\ell m n}\right\} z_{a}\right]}{2 \alpha-i \Delta B_{\ell m n}} \\
& \times \frac{1-\exp \left[-\left\{2 \alpha+i \Delta B_{\ell^{\prime} m^{\prime} n}\right\} z_{a}\right]}{2 \alpha+i \Delta B_{\ell^{\prime} m^{\prime} n}} \\
& =\left(\frac{\gamma}{\pi}\right)^{2} \Omega_{0}^{3} \sum_{\ell} \sum_{m} \\
& G_{T_{x}}\left(\ell \Omega_{0}\right) G_{T_{x}}\left(m \Omega_{0}\right) G_{T_{x}}\left[(\ell+m-n) \Omega_{0}\right] \\
& \times \frac{1-2 \exp \left(-2 \alpha z_{a}\right) \cos \left(\Delta B_{\ell m n} z_{a}\right)+\exp \left(-4 \alpha z_{a}\right)}{4 \alpha^{2}+\left(\Delta B_{\ell m n}\right)^{2}} .
\end{aligned}
$$

In the above, a single polarized electric field has been considered. Considering dual polarized field [22]-[24], we have

$$
\begin{aligned}
& \widehat{P}_{\mathrm{NLI}}^{(\mathrm{ss})}\left(z_{a}, n \Omega_{0}\right)=\frac{8}{27}\left\langle\left|\widehat{E}_{\mathrm{NLI}}^{(\mathrm{ss})}\left(z_{a}, n \Omega_{0}\right)\right|^{2}\right\rangle \\
& =\frac{8}{27}\left(\frac{\gamma}{2 \pi}\right)^{2} \Omega_{0}^{3} \sum_{\ell} \sum_{m} \\
& G_{T_{x}}\left(\ell \Omega_{0}\right) G_{T_{x}}\left(m \Omega_{0}\right) G_{T_{x}}\left[(\ell+m-n) \Omega_{0}\right] \\
& \times \frac{1-2 \exp \left(-2 \alpha z_{a}\right) \cos \left(\Delta B_{\ell m n} z_{a}\right)+\exp \left(-4 \alpha z_{a}\right)}{4 \alpha^{2}+\left(\Delta B_{\ell m n}\right)^{2}} .
\end{aligned}
$$

Therefore, the NLI power at the output end of the transmission line, $\widehat{P}_{\mathrm{NLI}}\left(N z_{a}, n \Omega_{0}\right)$ is represented as

$$
\begin{aligned}
& \widehat{P}_{\mathrm{NLI}}\left(N z_{a}, n \Omega_{0}\right)=N \widehat{P}_{\mathrm{NLI}}^{(\mathrm{ss})}\left(z_{a}, n \Omega_{0}\right) \\
& =\frac{2}{27}\left(\frac{\gamma}{\pi}\right)^{2} N \Omega_{0}^{3} \sum_{\ell} \sum_{m} \\
& G_{T_{x}}\left(\ell \Omega_{0}\right) G_{T_{x}}\left(m \Omega_{0}\right) G_{T_{x}}\left[(\ell+m-n) \Omega_{0}\right] \\
& \times \frac{1-2 \exp \left(-2 \alpha z_{a}\right) \cos \left(\Delta B_{\ell m n} z_{a}\right)+\exp \left(-4 \alpha z_{a}\right)}{4 \alpha^{2}+\left(\Delta B_{\ell m n}\right)^{2}} .
\end{aligned}
$$

\subsubsection{Gaussian Noise Model for Coherent FWM Light}

When the FWM light generated in each span is coherent, the total nonlinear interference light at the output end $z=N z_{a}$, 
$\widehat{E}_{\mathrm{NLI}}^{\text {(total) }}\left(N z_{a}, \Delta \omega\right)$ can be represented as

$$
\begin{aligned}
& \widehat{E}_{\mathrm{NLI}}^{\text {(total) }}\left(N z_{a}, \Delta \omega\right) \\
& =\sum_{n=-\infty}^{\infty} \widehat{E}_{\mathrm{NLI}}^{\text {(total }}\left(N z_{a}, n \Omega_{0}\right) \delta\left(\Delta \omega-n \Omega_{0}\right) .
\end{aligned}
$$

$\widehat{E}_{\mathrm{NLI}}^{\text {(total) }}\left(N z_{a}, n \Omega_{0}\right)$ can be calculated by using Eq. $(85)$ as

$$
\begin{aligned}
& \widehat{E}_{\mathrm{NLI}}^{\text {(total) }}\left(N z_{a}, n \Omega_{0}\right)=\sum_{\ell} \sum_{m} \widehat{E}^{\text {(total) }}\left(N z_{a}, n \Omega_{0}\right) \\
& =i \frac{\gamma}{2 \pi} \Omega_{0}^{3 / 2} \exp \left[i \Delta \beta\left(n \Omega_{0}\right) N z_{a}\right] \sum_{\ell} \sum_{m} \xi_{\ell} \xi_{m} \xi_{\ell+m-n}^{*} \\
& \times \sqrt{G_{T_{x}}\left(\ell \Omega_{0}\right) G_{T_{x}}\left(m \Omega_{0}\right) G_{T_{x}}\left[(\ell+m-n) \Omega_{0}\right]} \\
& \times \frac{1-\exp \left[-\left(2 \alpha-i \Delta B_{\ell m n}\right) z_{a}\right]}{2 \alpha-i \Delta B_{\ell m n}} \\
& \times \frac{\sin \left(\frac{\Delta B_{\ell m n} N z_{a}}{2}\right)}{\sin \left(\frac{\Delta B_{\ell m n} z_{a}}{2}\right)} \exp \left[i \frac{\Delta B_{\ell m n}(N-1) z_{a}}{2}\right] .
\end{aligned}
$$

Therefore the NLI power at the output end of the transmission line, $\widehat{P}_{\mathrm{NLI}}^{\text {(total) }}\left(N z_{a}, n \Omega_{0}\right)$, is represented as

$$
\begin{aligned}
& \widehat{P}_{\mathrm{NLI}}^{\text {total }}\left(N z_{a}, n \Omega_{0}\right)=\left\langle\left|\widehat{E}_{\mathrm{NLI}}^{\text {(total) }}\left(N z_{a}, n \Omega_{0}\right)\right|^{2}\right\rangle \\
& =\left\langle\left(\frac{\gamma}{2 \pi}\right)^{2} \Omega_{0}^{3} \sum_{\ell} \sum_{m} \xi_{\ell} \xi_{m} \xi_{\ell+m-n}^{*}\right. \\
& \times \sqrt{G_{T_{x}}\left(\ell \Omega_{0}\right) G_{T_{x}}\left(m \Omega_{0}\right) G_{T_{x}}\left[(\ell+m-n) \Omega_{0}\right]} \\
& \times \frac{1-\exp \left[-\left(2 \alpha-i \Delta B_{\ell m n}\right) z_{a}\right]}{2 \alpha-i \Delta B_{\ell m n}} \\
& \times \frac{\sin \left(\frac{\Delta B_{\ell m n} N z_{a}}{2}\right)}{\sin \left(\frac{\Delta B_{\ell m n} z_{a}}{2}\right)} \exp \left[i \frac{\Delta B_{\ell m n}(N-1) z_{a}}{2}\right] \\
& \times \sum_{\ell^{\prime}} \sum_{m^{\prime}} \xi_{\ell^{\prime}}^{*} \xi_{m^{\prime}}^{*} \xi_{\ell^{\prime}+m^{\prime}-n} \\
& \times \sqrt{G_{T_{x}}\left(\ell^{\prime} \Omega_{0}\right) G_{T_{x}}\left(m^{\prime} \Omega_{0}\right) G_{T_{x}}\left[\left(\ell^{\prime}+m^{\prime}-n\right) \Omega_{0}\right]} \\
& \times \frac{1-\exp \left[-\left(2 \alpha+i \Delta B_{\ell^{\prime} m^{\prime} n}\right) z_{a}\right]}{2 \alpha+i \Delta B_{\ell^{\prime} m^{\prime} n}} \\
& \left.\times \frac{\sin \left(\frac{\Delta B_{\ell^{\prime} m^{\prime} n} N z_{a}}{2}\right)}{\sin \left(\frac{\Delta B_{\ell^{\prime} m^{\prime} n} z_{a}}{2}\right)} \exp \left[-i \frac{\Delta B_{\ell^{\prime} m^{\prime} n}(N-1) z_{a}}{2}\right]\right\rangle \\
& =\left(\frac{\gamma}{2 \pi}\right)^{2} \Omega_{0}^{3} \sum_{\ell} \sum_{m} \sum_{\ell^{\prime}} \sum_{m^{\prime}} \\
& \left\langle\xi_{\ell} \xi_{m} \xi_{\ell+m-n}^{*} \xi_{\ell^{\prime}}^{*} \xi_{m^{\prime}}^{*} \xi_{\ell^{\prime}+m^{\prime}-n}\right\rangle \\
& \times \sqrt{G_{T_{x}}\left(\ell \Omega_{0}\right) G_{T_{x}}\left(m \Omega_{0}\right) G_{T_{x}}\left[(\ell+m-n) \Omega_{0}\right]} \\
& \times \sqrt{G_{T_{x}}\left(\ell^{\prime} \Omega_{0}\right) G_{T_{x}}\left(m^{\prime} \Omega_{0}\right) G_{T_{x}}\left[\left(\ell^{\prime}+m^{\prime}-n\right) \Omega_{0}\right]}
\end{aligned}
$$

$$
\begin{aligned}
& \times \frac{1-\exp \left[-\left(2 \alpha-i \Delta B_{\ell m n}\right) z_{a}\right]}{2 \alpha-i \Delta B_{\ell m n}} \\
& \times \frac{1-\exp \left[-\left(2 \alpha+i \Delta B_{\ell^{\prime} m^{\prime} n}\right) z_{a}\right]}{2 \alpha+i \Delta B_{\ell^{\prime} m^{\prime} n}} \\
& \times \frac{\sin \left(\frac{\Delta B_{\ell m n} N z_{a}}{2}\right)}{\sin \left(\frac{\Delta B_{\ell m n} z_{a}}{2}\right)} \frac{\sin \left(\frac{\Delta B_{\ell^{\prime} m^{\prime} n} N z_{a}}{2}\right)}{\sin \left(\frac{\Delta B_{\ell^{\prime} m^{\prime} n} z_{a}}{2}\right)} \\
& \times \exp \left[i \frac{\Delta B_{\ell m n}(N-1) z_{a}}{2}\right] \\
& \times \exp \left[-i \frac{\Delta B_{\ell^{\prime} m^{\prime} n}(N-1) z_{a}}{2}\right] \\
& =\left(\frac{\gamma}{2 \pi}\right)^{2} \Omega_{0}^{3} \sum_{\ell} \sum_{\ell^{\prime}}\left\langle\xi_{\ell} \xi_{\ell^{\prime}}^{*}\right\rangle \\
& \times \sum_{m} \sum_{m^{\prime}}\left\langle\xi_{m} \xi_{m^{\prime}}^{*}\right\rangle\left\langle\xi_{\ell+m-n}^{*} \xi_{\ell^{\prime}+m^{\prime}-n}\right\rangle \\
& \times \sqrt{G_{T_{x}}\left(\ell \Omega_{0}\right) G_{T_{x}}\left(m \Omega_{0}\right) G_{T_{x}}\left[(\ell+m-n) \Omega_{0}\right]} \\
& \times \sqrt{G_{T_{x}}\left(\ell^{\prime} \Omega_{0}\right) G_{T_{x}}\left(m^{\prime} \Omega_{0}\right) G_{T_{x}}\left[\left(\ell^{\prime}+m^{\prime}-n\right) \Omega_{0}\right]} \\
& \times \frac{1-\exp \left[-\left(2 \alpha-i \Delta B_{\ell m n}\right) z_{a}\right]}{2 \alpha-i \Delta B_{\ell m n}} \\
& \times \frac{1-\exp \left[-\left(2 \alpha+i \Delta B_{\ell^{\prime} m^{\prime} n}\right) z_{a}\right]}{2 \alpha+i \Delta B_{\ell^{\prime} m^{\prime} n}} \\
& \times \frac{\sin \left(\frac{\Delta B_{\ell m n} N z_{a}}{2}\right)}{\sin \left(\frac{\Delta B_{\ell m n} z_{a}}{2}\right)} \frac{\sin \left(\frac{\Delta B_{\ell^{\prime} m^{\prime} n} N z_{a}}{2}\right)}{\sin \left(\frac{\Delta B_{\ell^{\prime} m^{\prime} n} z_{a}}{2}\right)} \\
& \times \exp \left[i \frac{\Delta B_{\ell m n}(N-1) z_{a}}{2}\right] \\
& \times \exp \left[-i \frac{\Delta B_{\ell^{\prime} m^{\prime} n}(N-1) z_{a}}{2}\right] \\
& =\left(\frac{\gamma}{2 \pi}\right)^{2} \Omega_{0}^{3} \sum_{\ell} \sum_{m} \\
& G_{T_{x}}\left(\ell \Omega_{0}\right) G_{T_{x}}\left(m \Omega_{0}\right) G_{T_{x}}\left[(\ell+m-n) \Omega_{0}\right] \\
& \times \frac{1-2 \exp \left(-2 \alpha z_{a}\right) \cos \left(\Delta B_{\ell m n} z_{a}\right)+\exp \left(-4 \alpha z_{a}\right)}{4 \alpha^{2}+\left(\Delta B_{\ell m n}\right)^{2}} \\
& \times \frac{\sin ^{2}\left(\frac{\Delta B_{\ell m n} N z_{a}}{2}\right)}{\sin ^{2}\left(\frac{\Delta B_{\ell m n} z_{a}}{2}\right)} \text {. }
\end{aligned}
$$

For the dual polarized field, we have

$$
\begin{aligned}
& \widehat{P}_{\mathrm{NLI}}^{\text {total }}\left(N z_{a}, n \Omega_{0}\right)=\frac{8}{27}\left\langle\left|\widehat{E}_{\mathrm{NLI}}^{\text {total }}\left(N z_{a}, n \Omega_{0}\right)\right|^{2}\right\rangle \\
& =\frac{2}{27}\left(\frac{\gamma}{\pi}\right)^{2} \Omega_{0}^{3} \sum_{\ell} \sum_{m} G_{T_{x}}\left(\ell \Omega_{0}\right) G_{T_{x}}\left(m \Omega_{0}\right) \\
& \times G_{T_{x}}\left[(\ell+m-n) \Omega_{0}\right] \\
& \times \frac{1-2 \exp \left(-2 \alpha z_{a}\right) \cos \left(\Delta B_{\ell m n} z_{a}\right)+\exp \left(-4 \alpha z_{a}\right)}{4 \alpha^{2}+\left(\Delta B_{\ell m n}\right)^{2}}
\end{aligned}
$$




$$
\times \frac{\sin ^{2}\left(\frac{\Delta B_{\ell m n} N z_{a}}{2}\right)}{\sin ^{2}\left(\frac{\Delta B_{\ell m n} z_{a}}{2}\right)} .
$$

3.5 Power Spectral Density of Nonlinear Interference Noise

Since the following expression is satisfied for any function of $\widehat{f}(\Delta \omega)$,

$$
\int_{-\infty}^{\infty} \widehat{f}(\Delta \omega) d(\Delta \omega)=\lim _{\Omega_{0} \rightarrow 0} \sum_{n=-\infty}^{\infty} \Omega_{0} \widehat{f}\left(n \Omega_{0}\right),
$$

by setting $\Omega_{0} \rightarrow 0\left(T_{0} \rightarrow \infty\right)$ in Eq. (90), $\ell \Omega_{0}=\Delta \omega_{1}$, $m \Omega_{0}=\Delta \omega_{2}$, and $n \Omega_{0}=\Delta \omega$, we have

$$
\begin{aligned}
& G_{\mathrm{NLI}}\left(N z_{a}, \Delta \omega\right)=\lim _{T_{0} \rightarrow \infty} \Omega_{0} \widehat{P}_{\mathrm{NLI}}\left(N z_{a}, n \Omega_{0}\right) \\
& =\frac{2}{27}\left(\frac{\gamma}{\pi}\right)^{2} N \int_{-\infty}^{\infty} \int_{-\infty}^{\infty} G_{T_{x}}\left(\Delta \omega_{1}\right) G_{T_{x}}\left(\Delta \omega_{2}\right) \\
& \times G_{T_{x}}\left(\Delta \omega_{1}+\Delta \omega_{2}-\Delta \omega\right) \\
& \times \frac{1-2 \exp \left(-2 \alpha z_{a}\right) \cos \left(\Delta B z_{a}\right)+\exp \left(-4 \alpha z_{a}\right)}{4 \alpha^{2}+(\Delta B)^{2}} \\
& \times d\left(\Delta \omega_{1}\right) d\left(\Delta \omega_{2}\right) .
\end{aligned}
$$

From Eq. (94), we also have

$$
\begin{aligned}
& G_{\mathrm{NLI}}^{\text {(toatl }}\left(N z_{a}, \Delta \omega\right)=\lim _{T_{0} \rightarrow \infty} \Omega_{0} \widehat{P}_{\mathrm{NLI}}^{\text {total }}\left(N z_{a}, n \Omega_{0}\right) \\
& =\frac{2}{27}\left(\frac{\gamma}{\pi}\right)^{2} \int_{-\infty}^{\infty} \int_{-\infty}^{\infty} G_{T_{x}}\left(\Delta \omega_{1}\right) G_{T_{x}}\left(\Delta \omega_{2}\right) \\
& \times G_{T_{x}}\left(\Delta \omega_{1}+\Delta \omega_{2}-\Delta \omega\right) \\
& \times \frac{1-2 \exp \left(-2 \alpha z_{a}\right) \cos \left(\Delta B z_{a}\right)+\exp \left(-4 \alpha z_{a}\right)}{4 \alpha^{2}+(\Delta B)^{2}} \\
& \times \frac{\sin ^{2}\left(\frac{\Delta B N z_{a}}{2}\right)}{\sin ^{2}\left(\frac{\Delta B z_{a}}{2}\right)} d\left(\Delta \omega_{1}\right) d\left(\Delta \omega_{2}\right) .
\end{aligned}
$$

$\Delta B$ in Eqs. (96) and (97), is given by a formula in which $\Delta \omega_{4}$ in Eq. (75) is replaced with $\Delta \omega$.

\subsection{Nyquist Limit}

Here we focus on a Nyquist WDM transmission system. In this case, the symbol rate $R_{S}$ is equal to the channel bandwidth $B_{\mathrm{ch}}$. We assume that $G_{T_{x}}(\Delta \omega)$ is given by

$$
G_{T_{x}}(\Delta \omega)= \begin{cases}G_{\mathrm{WDM}} & |\Delta \omega| \leq \pi B_{\mathrm{WDM}}, \\ 0 & \text { otherwise } .\end{cases}
$$

Here, $G_{\mathrm{WDM}}$ and $B_{\mathrm{WDM}}$ represent the transmission power spectral density and total bandwidth of the Nyquist WDM signal, respectively. By setting $\Delta \omega=0$ in Eq. (96), we have

$$
\begin{aligned}
& G_{\mathrm{NLI}}\left(N z_{a}, 0\right) \\
& =\frac{2}{27}\left(\frac{\gamma}{\pi}\right)^{2} N G_{\mathrm{wDM}}^{3} \iint_{\mathcal{D}}\left[1-2 \exp \left(-2 \alpha z_{a}\right)\right.
\end{aligned}
$$

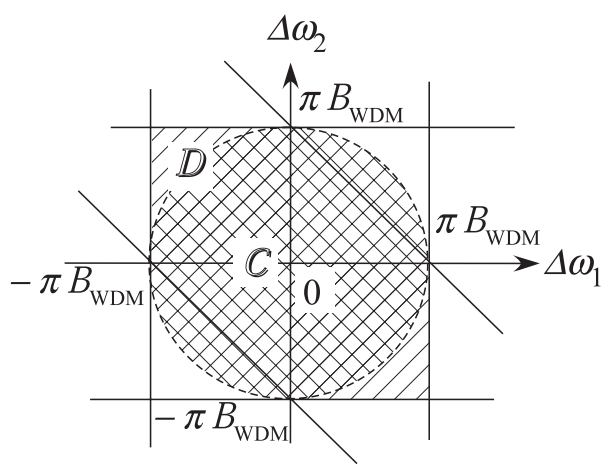

Fig. 2 The domain of integration $\mathcal{D}$ and approximated domain of integration $C$.

$$
\begin{aligned}
& \left.\times \cos \left\{\left|\beta_{0}^{(2)}\right|\left(\Delta \omega_{1}\right)\left(\Delta \omega_{2}\right) z_{a}\right\}+\exp \left(-4 \alpha z_{a}\right)\right] \\
& \times\left[4 \alpha^{2}+\left\{\left|\beta_{0}^{(2)}\right|\left(\Delta \omega_{1}\right)\left(\Delta \omega_{2}\right)\right\}^{2}\right]^{-1} d\left(\Delta \omega_{1}\right) d\left(\Delta \omega_{2}\right) .
\end{aligned}
$$

Here, we assume the third order dispersion $\beta_{0}^{(3)}=0$ in Eq. (75). In this case, the domain of integration $\mathcal{D}$ in the $\Delta \omega_{1}-\Delta \omega_{2}$ plane is determined by Eq. (98) and it satisfies

$$
\left|\Delta \omega_{1}\right|,\left|\Delta \omega_{2}\right|,\left|\Delta \omega_{1}+\Delta \omega_{2}\right| \leq \pi B_{\mathrm{WDM}} .
$$

The domain of integration $\mathcal{D}$ is shown in Fig. 2. Analytical integration in the domain of $\mathcal{D}$ is difficult. Then the domain of integration is approximated by a circular domain $C$ with the radius of $\pi B_{\text {WDM }}$ which is surrounded by a dotted line in Fig. 2. The numerator of the integrand is also approximated as

$$
\begin{aligned}
& 1-2 \exp \left(-2 \alpha z_{a}\right) \cos \left\{\left|\beta_{0}^{(2)}\right|\left(\Delta \omega_{1}\right)\left(\Delta \omega_{2}\right) z_{a}\right\} \\
& +\exp \left(-4 \alpha z_{a}\right) \\
& =1-2 \exp \left(-2 \alpha z_{a}\right) \\
& \times\left[1-2 \sin ^{2}\left\{\frac{\left|\beta_{0}^{(2)}\right|\left(\Delta \omega_{1}\right)\left(\Delta \omega_{2}\right) z_{a}}{2}\right\}\right] \\
& +\exp \left(-4 \alpha z_{a}\right) \\
& =1-2 \exp \left(-2 \alpha z_{a}\right)+\exp \left(-4 \alpha z_{a}\right) \\
& +4 \exp \left(-2 \alpha z_{a}\right) \sin ^{2}\left\{\frac{\left|\beta_{0}^{(2)}\right|\left(\Delta \omega_{1}\right)\left(\Delta \omega_{2}\right) z_{a}}{2}\right\} \\
& =\left\{1-\exp \left(-2 \alpha z_{a}\right)\right\}^{2} \\
& +4 \exp \left(-2 \alpha z_{a}\right) \sin ^{2}\left\{\frac{\left|\beta_{0}^{(2)}\right|\left(\Delta \omega_{1}\right)\left(\Delta \omega_{2}\right) z_{a}}{2}\right\} \\
& \simeq\left\{1-\exp \left(-2 \alpha z_{a}\right)\right\}^{2} .
\end{aligned}
$$

Furthermore, the effective length of optical amplifier spacing $L_{\text {eff }}$ and the asymptotic effective length $L_{\text {eff.a }}$ are respectively defined by

$$
\begin{aligned}
L_{\text {eff }} & =\int_{0}^{z_{a}} \exp (-2 \alpha z) d z \\
& =\frac{1-\exp \left(-2 \alpha z_{a}\right)}{2 \alpha}, \\
L_{\text {eff }, \mathrm{a}} & =\lim _{z_{a} \rightarrow \infty} L_{\text {eff }}=\frac{1}{2 \alpha} .
\end{aligned}
$$

Using Eqs. (101)-(103), Eq. (99) becomes 


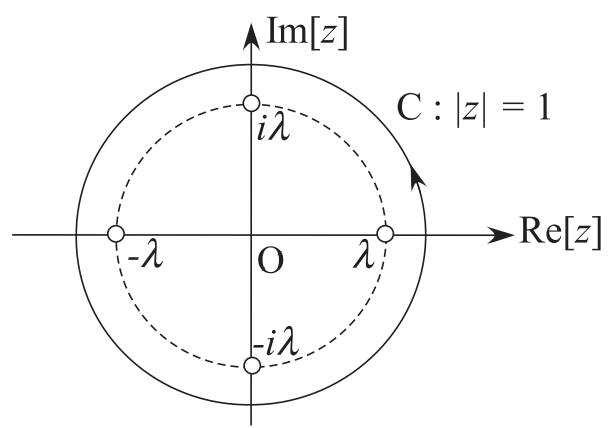

Fig.3 Integration on a unit circle $C$ and four simple poles.

$$
\begin{aligned}
& G_{\mathrm{NLI}}\left(N z_{a}, 0\right)=\frac{2}{27}\left(\frac{\gamma}{\pi}\right)^{2} N G_{\mathrm{wDM}}^{3} L_{\mathrm{eff}}^{2} \\
& \times \iint_{C} \frac{d\left(\Delta \omega_{1}\right) d\left(\Delta \omega_{2}\right)}{1+\left\{\left|\beta_{0}^{(2)}\right|\left(\Delta \omega_{1}\right)\left(\Delta \omega_{2}\right) L_{\mathrm{eff}, a}\right\}^{2}} .
\end{aligned}
$$

Next, the following variable transformation is introduced to integrate in the circular domain $C$. By setting

$$
\left\{\begin{array}{l}
\Delta \omega_{1}=r \cos \varphi \\
\Delta \omega_{2}=r \sin \varphi
\end{array}\right.
$$

we can rewrite Eq. (104) as

$$
\begin{aligned}
& G_{\mathrm{NLI}}\left(N z_{a}, 0\right)=\frac{2}{27}\left(\frac{\gamma}{\pi}\right)^{2} N G_{\mathrm{wDM}}^{3} L_{\mathrm{eff}}^{2} \\
& \times \int_{0}^{2 \pi} \int_{0}^{\pi B_{\mathrm{WDM}}} \frac{r d r d \varphi}{1+\left(\left|\beta_{0}^{(2)}\right| L_{\mathrm{eff}, \mathrm{a}}\right)^{2} r^{4} \cos ^{2} \varphi \sin ^{2} \varphi} .
\end{aligned}
$$

By setting $z=\exp (i \varphi)$ in Eq. (106), the integration with respect to $\varphi$ is replaced with the integration on a unit circle $C$ with $|z|=1$ which center is located at the origin of the complex plane shown in Fig. 3. Then we have

$$
\begin{aligned}
I_{\varphi}= & \int_{0}^{2 \pi} \frac{r}{1+\left(\left|\beta_{0}^{(2)}\right| L_{\text {eff, } \mathrm{a}}\right)^{2} r^{4} \cos ^{2} \varphi \sin ^{2} \varphi} d \varphi \\
= & r \int_{C} \frac{\left\{\frac{1}{2}\left(z+\frac{1}{z}\right)\right\}^{-2}\left\{\frac{1}{2 i}\left(z-\frac{1}{z}\right)\right\}^{-2}}{1+\left(\left|\beta_{0}^{(2)}\right| L_{\mathrm{eff}, \mathrm{r}} r^{2}\right)^{2}} \frac{d z}{i z} \\
= & i \frac{16 r}{\left(\left|\beta_{0}^{(2)}\right| L_{\mathrm{eff}, \mathrm{a}} r^{2}\right)^{2}} \\
& \times \int_{C} z^{3}\left(z^{4}+\frac{4}{\left|\beta_{0}^{(2)}\right| L_{\mathrm{eff}, \mathrm{a}} r^{2}} z^{2}-1\right)^{-1} \\
& \times\left(z^{4}-\frac{4}{\left|\beta_{0}^{(2)}\right| L_{\mathrm{eff}, \mathrm{a}} r^{2}} z^{2}-1\right)^{-1} d z .
\end{aligned}
$$

In this case, the integrand in Eq. (107) has four simple poles in the domain surrounded by $C$,

$$
\begin{aligned}
& z= \pm \lambda, \pm i \lambda, \\
& \lambda=\sqrt{\sqrt{\left(\frac{2}{\left|\beta_{0}^{(2)}\right| L_{\text {eff }, a} r^{2}}\right)^{2}+1-\frac{2}{\left|\beta_{0}^{(2)}\right| L_{\text {eff, } a} r^{2}}}} .
\end{aligned}
$$

Then Eq. (107) can be represented as

$$
I_{\varphi}=i \frac{16 r}{\left(\left|\beta_{0}^{(2)}\right| L_{\mathrm{eff}, \mathrm{a}} r^{2}\right)^{2}} \int_{C} f(z) d z,
$$

where

$$
f(z)=\frac{z^{3}(z-\lambda)^{-1}(z+\lambda)^{-1}(z-i \lambda)^{-1}(z+i \lambda)^{-1}}{z^{4}-\left\{\sqrt{\left(\frac{2}{\left|\beta_{0}^{(2)}\right| L_{\mathrm{eff}, a} r^{2}}\right)^{2}+1}+\frac{2}{\left|\beta_{0}^{(2)}\right| L_{\mathrm{eff}, \mathrm{a}}{ }^{2}}\right\}^{2}} .
$$

The complex integral of Eq. (109) becomes

$$
\begin{aligned}
& I_{\varphi}=i \frac{16 r}{\left(\left|\beta_{0}^{(2)}\right| L_{\text {eff,a }} r^{2}\right)^{2}} \times(2 i \pi) \\
& \times\{\operatorname{Res}[f(z), \lambda]+\operatorname{Res}[f(z),-\lambda] \\
& +\operatorname{Res}[f(z), i \lambda]+\operatorname{Res}[f(z),-i \lambda]\} .
\end{aligned}
$$

Here,

$$
\begin{aligned}
& \operatorname{Res}[f(z), \lambda]=\operatorname{Res}[f(z),-\lambda] \\
& =\operatorname{Res}[f(z), i \lambda]=\operatorname{Res}[f(z),-i \lambda] \\
& =\frac{\lambda^{3}(\lambda+\lambda)^{-1}(\lambda-i \lambda)^{-1}(\lambda+i \lambda)^{-1}}{\lambda^{4}-\left\{\sqrt{\left(\frac{2}{\left|\beta_{0}^{(2)}\right| L_{\mathrm{eff}, \mathrm{a}} r^{2}}\right)^{2}+1}+\frac{2}{\left|\beta_{0}^{(2)}\right| L_{\mathrm{eff}, \mathrm{a}} r^{2}}\right\}^{2}} \\
& =\frac{1}{4\left[\left\{\sqrt{\left(\frac{2}{\left|\beta_{0}^{(2)}\right| L_{\text {eff, } a} r^{2}}\right)^{2}+1}-\frac{2}{\left|\beta_{0}^{(2)}\right| L_{\text {eff, }, a} r^{2}}\right\}^{2}\right.} \\
& \left.-\left\{\sqrt{\left(\frac{2}{\left|\beta_{0}^{(2)}\right| L_{\text {eff }, a} r^{2}}\right)^{2}+1}+\frac{2}{\left|\beta_{0}^{(2)}\right| L_{\text {eff }, a} r^{2}}\right\}^{2}\right] \\
& =-\frac{\left|\beta_{0}^{(2)}\right| L_{\mathrm{eff}, \mathrm{a}} r^{2}}{32 \sqrt{\left(\frac{2}{\left|\beta_{0}^{(2)}\right| L_{\mathrm{eff}, \mathrm{r}} r^{2}}\right)^{2}+1}} \\
& =-\frac{\left(\left|\beta_{0}^{(2)}\right| L_{\mathrm{eff}, \mathrm{a}} r^{2}\right)^{2}}{64 \sqrt{1+\left(\frac{\left|\beta_{0}^{(2)}\right| L_{\mathrm{eff}, \mathrm{a}} r^{2}}{2}\right)^{2}}} .
\end{aligned}
$$

Therefore the complex integral of Eq. (109) leads

$$
I_{\varphi}=\frac{32 \pi r}{\left(\left|\beta_{0}^{(2)}\right| L_{\text {eff, }} r^{2}\right)^{2}} \times 4
$$




$$
\begin{gathered}
\times \frac{\left(\left|\beta_{0}^{(2)}\right| L_{\mathrm{eff}, \mathrm{a}} r^{2}\right)^{2}}{64 \sqrt{1+\left(\frac{\left|\beta_{0}^{(2)}\right| L_{\mathrm{eff}, \mathrm{a}} r^{2}}{2}\right)^{2}}} \\
=\frac{2 \pi r}{\sqrt{1+\left(\frac{\left|\beta_{0}^{(2)}\right| L_{\mathrm{eff}, \mathrm{a}} r^{2}}{2}\right)^{2}}} \cdot
\end{gathered}
$$

Substituting Eq. (113) into Eq. (106), we have

$$
\begin{aligned}
& G_{\mathrm{NLI}}\left(N z_{a}, 0\right)=\frac{2}{27}\left(\frac{\gamma}{\pi}\right)^{2} N G_{\mathrm{WDM}}^{3} L_{\mathrm{eff}}^{2} \\
& \sqrt{1+\left(\frac{\left|\beta_{0}^{(2)}\right| L_{\mathrm{eff}, a} r^{2}}{2}\right)^{2}}
\end{aligned} r .
$$

Furthermore, by changing variable with $R=\left|\beta_{0}^{(2)}\right| L_{\text {eff, }} r^{2} / 2$ and integrating it, we obtain

$$
\begin{aligned}
& G_{\mathrm{NII}}\left(N z_{a}, 0\right) \\
& =\frac{2}{27}\left(\frac{\gamma}{\pi}\right)^{2} N G_{\mathrm{wDM}}^{3} L_{\mathrm{eff}}^{2} \\
& \times \int_{0}^{\pi^{2}\left|\beta_{0}^{(2)}\right| L_{\mathrm{eff}, \mathrm{a}} B_{\mathrm{WDM}}^{2} / 2} \frac{2 \pi}{\sqrt{1+R^{2}}} \frac{d R}{\left|\beta_{0}^{(2)}\right| L_{\mathrm{eff}, \mathrm{a}}} \\
& =\frac{4}{27} \frac{\gamma^{2} N G_{\mathrm{WDM}}^{3} L_{\mathrm{eff}}^{2}}{\pi\left|\beta_{0}^{(2)}\right| L_{\mathrm{eff}, \mathrm{a}}}\left[\sinh ^{-1} R\right]_{0}^{\pi^{2}\left|\beta_{0}^{(2)}\right| L_{\mathrm{eff}, \mathrm{a}} B_{\mathrm{WDM}}^{2} / 2} \\
& =\frac{4}{27} \frac{\gamma^{2} N G_{\mathrm{wDM}}^{3} L_{\mathrm{eff}}^{2}}{\pi\left|\beta_{0}^{(2)}\right| L_{\mathrm{eff}, \mathrm{a}}} \sinh ^{-1}\left(\frac{\pi^{2}\left|\beta_{0}^{(2)}\right| L_{\mathrm{eff}, \mathrm{a}} B_{\mathrm{WDM}}^{2}}{2}\right) .
\end{aligned}
$$

For $x \gg 1$,

$$
\sinh ^{-1}(x)=\log _{e}\left(x+\sqrt{x^{2}+1}\right) \simeq \log _{e}(2 x) .
$$

Applying the approximation of Eq. (116) to Eq. (115), we obtain

$$
\begin{aligned}
& G_{\mathrm{NII}}\left(N z_{a}, 0\right) \\
& \simeq \frac{4}{27} \frac{\gamma^{2} N G_{\mathrm{wDM}}^{3} L_{\mathrm{eff}}^{2}}{\pi\left|\beta_{0}^{(2)}\right| L_{\text {eff,a }}} \log _{e}\left(\pi^{2}\left|\beta_{0}^{(2)}\right| L_{\mathrm{eff}, \mathrm{a}} B_{\mathrm{WDM}}^{2}\right) .
\end{aligned}
$$

The NLI noise power $P_{\mathrm{NLI}}$ can be calculated by using Eq. (38) as

$$
\begin{aligned}
& P_{\mathrm{NLI}}\left(N z_{a}\right) \\
& =\int_{-\infty}^{\infty} G_{\mathrm{NLI}}\left(N z_{a}, \Delta \omega\right)\left|H_{R_{x}}(\Delta \omega)\right|^{2} d(\Delta \omega) .
\end{aligned}
$$

By approximating $G_{\mathrm{NLI}}\left(N z_{a}, \Delta \omega\right) \simeq G_{\mathrm{NLI}}\left(N z_{a}, 0\right)$ and assuming the bandwidth of the receiver is equal to the OSNR bandwidth $B_{n}$, we set $H_{R_{x}}(\Delta \omega)$ as

$$
H_{R_{x}}(\Delta \omega)= \begin{cases}1 & |\Delta \omega| \leq \pi B_{n}, \\ 0 & \text { otherwise }\end{cases}
$$

Then we have

$$
\begin{aligned}
& P_{\mathrm{NLI}}\left(N z_{a}\right) \simeq 2 \pi B_{n} G_{\mathrm{NLI}}\left(N z_{a}, 0\right) \\
& =\frac{8}{27} \frac{\gamma^{2} N G_{\mathrm{WDM}}^{3} L_{\mathrm{eff}}^{2} B_{n}}{\left|\beta_{0}^{(2)}\right| L_{\mathrm{eff}, \mathrm{a}}} \log _{e}\left(\pi^{2}\left|\beta_{0}^{(2)}\right| L_{\mathrm{eff}, \mathrm{a}} B_{\mathrm{wDM}}^{2}\right) .
\end{aligned}
$$

By setting $L_{\text {eff,a }} \sim L_{\text {eff }}$, we obtain

$$
\begin{aligned}
P_{\mathrm{NLI}}\left(N z_{a}\right) \simeq & \left(\frac{2}{3}\right)^{3} \frac{\gamma^{2} N G_{\mathrm{wDM}}^{3} L_{\mathrm{eff}} B_{n}}{\left|\beta_{0}^{(2)}\right|} \\
& \times \log _{e}\left(\pi^{2}\left|\beta_{0}^{(2)}\right| L_{\mathrm{eff}} B_{\mathrm{WDM}}^{2}\right) .
\end{aligned}
$$

The total bandwidth of WDM signal $B_{\mathrm{WDM}}$, the transmission power spectral density $G_{\mathrm{WDM}}$, and the total transmission power $P_{\text {WDM }}$ are respectively expressed by using the total channel number $N_{\mathrm{ch}}$ and transmission power per channel $P_{\mathrm{ch}}$ as

$$
\begin{aligned}
B_{\mathrm{wDM}} & =N_{\mathrm{ch}} B_{\mathrm{ch}}=N_{\mathrm{ch}} R_{S}, \\
G_{\mathrm{wDM}} & =\frac{P_{\mathrm{ch}}}{B_{\mathrm{ch}}}=\frac{P_{\mathrm{ch}}}{R_{S}}, \\
P_{\mathrm{wDM}} & =N_{\mathrm{ch}} P_{\mathrm{ch}}=G_{\mathrm{WDM}} B_{\mathrm{WDM}} .
\end{aligned}
$$

By substituting these expressions into Eq. (121), we have

$$
\begin{aligned}
P_{\mathrm{NLI}}\left(N z_{a}\right)= & \left(\frac{2}{3}\right)^{3} \frac{\gamma^{2} N P_{\mathrm{ch}}^{3} L_{\mathrm{eff}} B_{n}}{\left|\beta_{0}^{(2)}\right| R_{s}^{3}} \\
& \times \log _{e}\left(\pi^{2}\left|\beta_{0}^{(2)}\right| L_{\mathrm{eff}} N_{\mathrm{ch}}^{2} R_{s}^{2}\right) .
\end{aligned}
$$

\section{Application to Evaluation and Design a Transmission System}

Let us consider to apply Eq. (125) to evaluate and design a Nyquist WDM transmission system. By setting $R_{s}=B_{n}$ in Eq. (39), Eq. (37) becomes

$$
\mathrm{OSNR}_{\mathrm{NL}}=\mathrm{SNR}_{\mathrm{NL}}=\frac{P_{\mathrm{ch}}}{P_{\mathrm{ASE}}+P_{\mathrm{NLI}}} .
$$

By defining a parameter $F$ which is independent of fiber characteristics such as noise figure of optical amplifier, the total ASE noise power after propagation through the total length of the transmission line $N z_{a}$ is represented as

$$
P_{\mathrm{ASE}}\left(N z_{a}\right)=F N G=F N \exp \left(2 \alpha z_{a}\right) .
$$

On the other hand, the NLI noise power can be represented by using Eq. (125) as

$$
P_{\mathrm{NLI}}\left(N z_{a}\right)=\eta \frac{\gamma^{2} N P_{\mathrm{ch}}^{3} L_{\mathrm{eff}}}{\left|\beta_{0}^{(2)}\right|}=h P_{\mathrm{ch}}^{3},
$$

where

$$
\eta=\left(\frac{2}{3}\right)^{3} \frac{\log _{e}\left(\pi^{2}\left|\beta_{0}^{(2)}\right| L_{\mathrm{eff}} N_{\mathrm{ch}}^{2} R_{s}^{2}\right)}{R_{S}^{2}}
$$




$$
h=\eta \frac{\gamma^{2} N L_{\mathrm{eff}}}{\left|\beta_{0}^{(2)}\right|}
$$

For simplicity, hereafter we treat $\eta$ as a constant. By substituting Eq. (128) into Eq. (126), we have

$$
\mathrm{OSNR}_{\mathrm{NL}}=\frac{P_{\mathrm{ch}}}{P_{\mathrm{ASE}}+h P_{\mathrm{ch}}^{3}} .
$$

Differentiating Eq. (131) with respect to $P_{\mathrm{ch}}$, we have

$$
\frac{\partial\left(\mathrm{OSNR}_{\mathrm{NL}}\right)}{\partial P_{\mathrm{ch}}}=\frac{P_{\mathrm{ASE}}-2 h P_{\mathrm{ch}}^{3}}{\left(P_{\mathrm{ASE}}+h P_{\mathrm{ch}}^{3}\right)^{2}} .
$$

By setting the right hand side of Eq. (132) to 0, the optimum value of $P_{\mathrm{ch}}$, i.e. $P_{\mathrm{ch}}^{(\mathrm{opt})}$ can be determined. By using Eqs. (127) and (130), we have

$$
\begin{aligned}
P_{\mathrm{ch}}^{\text {(opt) }} & =\left(\frac{P_{\mathrm{ASE}}}{2 h}\right)^{1 / 3} \\
& =\left(\frac{F}{2 \eta}\right)^{1 / 3}\left\{\frac{\left|\beta_{0}^{(2)}\right| \exp \left(2 \alpha z_{a}\right)}{\gamma^{2} L_{\mathrm{eff}}}\right\}^{1 / 3} .
\end{aligned}
$$

By representing $P_{\mathrm{ch}}^{\text {(opt) }}$ in Eq. (133) with the unit of $[\mathrm{dBm}]$, we have

$$
\begin{aligned}
& P_{\mathrm{ch}}^{\text {(opt) }}[\mathrm{dBm}]=10 \log _{10} P_{\mathrm{ch}}^{\text {(opt) }}[\mathrm{mW}] \\
& =C_{1}-\frac{10}{3} \log _{10}\left(\frac{\gamma^{2} L_{\mathrm{eff}}}{\left|\beta_{0}^{(2)}\right|}\right)+\frac{1}{3} \widehat{\alpha} z_{a} .
\end{aligned}
$$

Here, Eq. (29) was used to derive the above expression. $C_{1}$ is given by

$$
C_{1}=\frac{10}{3} \log _{10}\left(\frac{F}{2 \eta}\right),
$$

and it is independent of the transmission fiber. By substituting Eq. (133) into Eq. (131) and using Eqs. (127) and (130), we find the maximum value of $\mathrm{OSNR}_{\mathrm{NL}}$,

$$
\begin{aligned}
& \operatorname{OSNR}_{\mathrm{NL}}^{(\max )}=\frac{1}{3 h\left\{P_{\mathrm{ch}}^{(\text {opt }}\right\}^{2}} \\
& =\left(\frac{4}{27 F^{2} \eta}\right)^{1 / 3} \frac{1}{N}\left\{\frac{\left|\beta_{0}^{(2)}\right| \exp \left(-4 \alpha z_{a}\right)}{\gamma^{2} L_{\mathrm{eff}}}\right\}^{1 / 3} .
\end{aligned}
$$

By representing $\operatorname{OSNR}_{\mathrm{NL}}^{(\max )}$ with the unit of decibel, we obtain

$$
\begin{aligned}
& \operatorname{OSNR}_{\mathrm{NL}}^{(\max )}[\mathrm{dB}]=10 \log _{10} \operatorname{OSNR}_{\mathrm{NL}}^{(\max )} \\
& =C_{2}-\frac{10}{3} \log _{10}\left(\frac{\gamma^{2} L_{\mathrm{eff}}}{\left|\beta_{0}^{(2)}\right|}\right)-\frac{2}{3} \widehat{\alpha} z_{a}-10 \log _{10} N,
\end{aligned}
$$

where

$$
C_{2}=\frac{10}{3} \log _{10}\left(\frac{4}{27 F^{2} \eta}\right),
$$

and $C_{2}$ is independent of the transmission fiber, too.

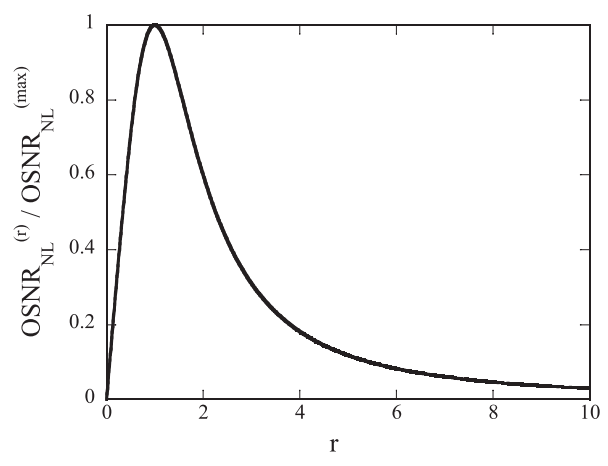

Fig. 4 Variation of $O S N R_{\mathrm{NL}}^{(\mathrm{r})}$ for $P_{\mathrm{ch}}=r P_{\mathrm{ch}}^{(\mathrm{opt})}$.

Next, we consider the relation between $\mathrm{OSNR}_{\mathrm{NL}}$ and $P_{\mathrm{ch}}$ when the transmission power per channel $P_{\mathrm{ch}}$ is not optimum. By setting $P_{\mathrm{ch}}=r P_{\mathrm{ch}}^{(\mathrm{opt})}$ with a constant $r, \mathrm{OSNR}_{\mathrm{NL}}^{(\mathrm{r})}$ can be represented by using Eqs. (131), (133), and (136) as

$$
\begin{aligned}
\operatorname{OSNR}_{\mathrm{NL}}^{(\mathrm{r})} & =\frac{r P_{\mathrm{ch}}^{(\mathrm{opt})}}{P_{\mathrm{ASE}}+h r^{3}\left(P_{\mathrm{ch}}^{(\mathrm{opt})}\right)^{3}} \\
& =\frac{3 r}{r^{3}+2} \operatorname{OSNR}_{\mathrm{NL}}^{(\max )} .
\end{aligned}
$$

By representing OSNR $\mathrm{NL}_{\mathrm{NL}}^{(\mathrm{r})}$ with the unit of decibell, we find

$$
\begin{aligned}
& \operatorname{OSNR}_{\mathrm{NL}}^{(\mathrm{r})}[\mathrm{dB}]=10 \log _{10} \operatorname{OSNR}_{\mathrm{NL}}^{(\mathrm{r})} \\
& =10 \log _{10}\left(\frac{3 r}{r^{3}+2}\right)+\operatorname{OSNR}_{\mathrm{NL}}^{\max }[\mathrm{dB}] .
\end{aligned}
$$

We show Eq. (139) in Fig. 4. The optimum transmission optical power $P_{\mathrm{ch}}^{\text {(opt) }}$ and the maximum value of OSNR, $\mathrm{OSNR}_{\mathrm{NL}}^{\text {(max) }}$, can be obtained in Eqs. (134) and (137), by the parameters of the transmmission line except $C_{1}$ in Eq. (135) and $C_{2}$ in Eq. (138), i.e., only by the second order dispersion coefficient $\beta_{0}^{(2)}$, nonlinear coefficient $\gamma$, loss coefficient $\alpha$, optical amplifier spacing $z_{a}$, number of sections $N$. The OSNR when the transmission optical power per channel $P_{\mathrm{ch}}$ deviates from its optimal value can be calculated by using Eq. (140). These results can be applied to evaluate and design a Nyquist WDM transmission system.

$\mathrm{SNR}_{\mathrm{NL}}$ is equal to $\mathrm{OSNR}_{\mathrm{NL}}$ in Nyquist WDM transmission system as shown in Eq. (126) and then $\mathrm{OSNR}_{\mathrm{NL}}^{\text {(max) }}$ in Eq. (136) gives the upper limit of the spectral efficiency shown in Eq. (1). This is the nonlinear Shannon limit.

\section{Conclusion}

The nonlinear Shannon limit has been considered as a kind of theoretical limitation. To clarify its meanings and applicable range, some fundamental papers on the nonlinear Shannon limit have been reviewed. The Gaussian noise model on the nonlinear Shannon limit has been experimentally validated for various fiber types in [8]. But it does not give the theoretical limitation of the channel capacity. Recently, various attempts to break the limitation by nonlinear compensation schemes and nonlinear transmission technologies have been reported [25]-[48]. The nonlinear Shannon limit 
is a transitional limitation which can be overcome.

\section{References}

[1] C.E. Shannon, "A mathematical theory of communications," Bell System Tech. J., vol.27, no.3, pp.379-423, July 1948, vol.27, no.4, pp.623-656, Oct. 1948.

[2] E. Yamazaki, S. Yamanaka, Y. Kisaka, T. Nakagawa, K. Murata, E. Yoshida, T. Sakano, M. Tomizawa, Y. Miyamoto, S. Matsuoka, J. Matsui, A. Shibayama, J. Abe, Y. Nakamura, H. Noguchi, K. Fukuchi, H. Onaka, K. Fukumitsu, K. Komaki, O. Takeuchi, Y. Sakamoto, H. Nakashima, T. Mizuochi, K. Kubo, Y. Miyata, H. Nishimoto, S. Hirano, and K. Onohara, "Fast optical channel recovery in field demonstration of 100-Gbit/s Ethernet over OTN using real-time DSP," Opt. Exp., vol.19, no.14, pp.13179-13184, June 2011.

[3] K. Kikuchi, "Analyses of wavelength- and polarization-division multiplexed transmission characteristics of optical quadratureamplitude-modulation signals," Opt. Exp., vol.19, no.19, pp.1798517995, Aug. 2011.

[4] S.J. Savory, "Digital coherent optical receivers: Algorithms and subsystems (invited)," IEEE J. Sel. Topics Quantum Electron., vol.16, no.5, pp.1164-1179, Sept./Oct. 2010.

[5] P. Poggiolini, A. Carena, V. Curri, G. Bosco, and F. Forghieri, "Analytical modeling of nonlinear propagation in uncompensated optical transmission links," IEEE Photon. Technol. Lett., vol.23, no.11, pp.742-744, June 2011.

[6] A. Carena, V. Curri, G. Bosco, P. Poggiolini, and F. Forghieri, "Modeling of the impact of nonlinear propagation effects in uncompensated optical coherent transmission links," IEEE/OSA J. Lightw. Technol., vol.30, no.10, pp.1524-1539, May 2012.

[7] P. Poggiolini, "The GN model of non-linear propagation in uncompensated coherent optical systems," IEEE/OSA J. Lightw. Technol., vol.30, no.24, pp.3857-3879, Dec. 2012.

[8] A. Nespola, S. Straullu, A. Carena, G. Bosco, R. Cigliutti, P. Poggiolini, M. Hirano, Y. Yamamoto, T. Sasaki, J. Bauwelinck, K. Verheyen, and F. Forghieri, "GN-model validation over seven fiber types in uncompensated PM-16QAM Nyquist-WDM links," IEEE Photon. Technol. Lett., vol.26, no.2, pp.206-209, Jan. 2014.

[9] P.P. Mitra and J.B. Stark, "Nonlinear limits to the information capacity of optical fibre communications," Nature, vol.411, pp.1027-1030, June 2001

[10] J. Tang, "The channel capacity of a multispan DWDM system employing dispersive nonlinear optical fibers and an ideal coherent optical receiver," IEEE/OSA J. Lightw. Technol., vol.20, no.7, pp.10951101, July 2002.

[11] J. Tang, "A comparison study of the Shannon channel capacity of various nonlinear optical fibers," IEEE/OSA J. Lightwave Technol., vol.24, no.5, pp.2070-2075, May 2006.

[12] A.D. Ellis, J. Zhao, and D. Cotter, "Approaching the non-linear Shannon limit," IEEE/OSA J. Lightw. Technol., vol.28, no.4, pp.423433, Feb. 2010.

[13] P. Johannisson and M. Karlsson, "Perturbation analysis of nonlinear propagation in a strongly dispersive optical communication system," IEEE/OSA J. Lightw. Technol., vol.31, no.8, pp.1273-1282, April 2013.

[14] A. Mecozzi and R.-J. Essiambre, "Nonlinear Shannon limit in pseudolinear coherent systems," IEEE/OSA J. Lightw. Technol., vol.30, no.12, pp.2011-2024, June 2012.

[15] R.-J. Essiambre, G. Kramer, P.J. Winzer, G.J. Foschini, and B. Goebel, "Capacity limits of optical fiber networks," IEEE/OSA J. Lightw. Technol., vol.28, no.4, pp.662-701, Feb. 2010.

[16] P. Serena and A. Bononi, "An alternative approach to the Gaussian noise model and its system implications," IEEE/OSA J. Lightw. Technol., vol.31, no.22, pp.3489-3499, Nov. 2013

[17] A. Carena, G. Bosco, V. Curri, Y. Jiang, P. Poggiolini, and F. Forghieri, "EGN model of non-linear fiber propagation," Opt. Exp., vol.22, no.13, pp.16335-16362, Nov. 2013.

[18] G. Agrawal, Nonlinear Fiber Optics, Fifth ed., Academic Press, 2013.

[19] J.G. Proakis and M. Salehi, Digital Communications, Fifth ed., McGraw-Hill International Editions, 2008.

[20] K.O. Hill, D.C. Johnson, B.S. Kawasaki, and R.I. MacDonald, "CW three-wave mixing in single-mode optical fibers," J. Appl. Phys. vol.49, no.10, pp.88-93, Oct. 1978.

[21] K. Inoue and H. Toba, "Fiber four-wave mixing in multi-amplifier systems with nonuniform chromatic dispersion," IEEE/OSA J. Lightw. Technol., vol.33, no.1, pp.88-93, Jan. 1995.

[22] M. Matsumoto, Y. Akagi, and A. Hasegawa, "Propagation of solitons in fibers with randomly varying birefringence: Effects of soliton transmission control,” IEEE/OSA J. Lightw. Technol., vol.15, no.4, pp.584-589, April 1997.

[23] P.K.A. Wai, C.R. Menyuk, and H.H. Chen, "Stability of solitons in randomly varying birefringent fibers," Opt. Lett., vol.16, no.16, pp.1231-1233, Aug. 1991.

[24] C.R. Menyuk, "Nonlinear pulse propagation in birefringent optical fibers," IEEE J. Quantum Electron., vol.QE-23, no.2, pp.174-176, Feb. 1987.

[25] X. Li, X. Chen, G. Goldfarb, E. Mateo, I. Kim, F. Yaman, and G. Li, "Electronic post-compensation of WDM transmission impairments using coherent detection and digital signal processing," Opt. Exp., vol.16, no.2, pp.880-888, Jan. 2008.

[26] E. Ip and J.M. Kahn, "Compensation of dispersion and nonlinear impairments using digital backpropagation," IEEE/OSA J. Lightw. Technol., vol.26, no.20, pp.3416-3425, Oct. 2008.

[27] L. Liu, L. Li, Y. Huang, K. Cui, Q. Xiong, F.N. Hauske, C. Xie, Y. Cai, "Intrachannel nonlinearity compensation by inverse Volterra series transfer function," IEEE/OSA J. Lightw. Technol., vol.30, no.3, pp.310-316, Feb. 2012.

[28] W. Yan, Z. Tao, L. Dou, L. Li, S. Oda, T. Tanimura, T. Hoshida, and J.C. Rasmussen, "Low complexity digital perturbation backpropagation," The 37th European Conference and Exhibition on Optical Communication (ECOC2011), Geneva, Switzerland, Paper Tu.3.A.2, Sept. 2011.

[29] Z. Tao, L. Dou, W. Yan, L. Li, T. Hoshida, and J.C. Rasmussen, "Multiplier-free intrachannel nonlinearity compensating algorithm operating at symbol rate," IEEE/OSA J. Lightw. Technol., vol.29, no.17, pp.2570-2576, Sept. 2011.

[30] A. Hasegawa and T. Nyu, "Eigenvalue communication," IEEE/OSA J. Lightw. Technol., vol.11, no.3, pp.395-399, March 1993.

[31] H. Terauchi and A. Maruta, "Eigenvalue modulated optical transmission system based on digital coherent technology," The 10th Conference on Lasers and Electro-Optics Pacific Rim, and the 18th OptoElectronics and Communications Conference/Photonics in Switching 2013 (CLEO-PR OECC/PS 2013), Paper WR2-5, Kyoto, Japan, July 2013.

[32] H. Terauchi, Y. Matsuda, A. Toyota, and A. Maruta, "Noise tolerance of eigenvalue modulated optical transmission system based on digital coherent technology," The 19th OptoElectronics and Communications Conference/Australian Conference on Optical Fibre Technology 2014 (OECC/ACOFT 2014), Paper WEPS2-56, Melbourne, Australia July 2014.

[33] Y. Matsuda, H. Terauchi, and A. Maruta, "Design of eigenvaluemultiplexed multi-level modulation optical transmission system," The 19th OptoElectronics and Communications Conference/Australian Conference on Optical Fibre Technology 2014 (OECC/ACOFT 2014), Paper TH12B3, Melbourne, Australia, July 2014

[34] A. Maruta, "Eigenvalue modulated optical transmission system," The 20th OptoElectronics and Communications Conference 2015 (OECC 2015), Paper JThA.21, Shanghai, China, July 2015.

[35] A. Toyota and A. Maruta, "Wavelength division multiplexed optical eigenvalue modulated system," Tyrrhenian International Workshop on Digital Comminications 2015 (TIWDC 2015), Paper P3.4, Florence, Italy, Sept. 2015. 
[36] A. Maruta, A. Toyota, Y. Matsuda, and Y. Ikeda, "Experimental demonstration of long haul transmission of eigenvalue modulated signals," Tyrrhenian International Workshop on Digital Comminications 2015 (TIWDC 2015), Paper P2.6, Florence, Italy, Sept. 2015.

[37] A. Maruta and Y. Matsuda, "Polarization division multiplexed optical eigenvalue modulation," 2015 International Conference on Photonics in Switching (PS 2015), Paper FrII1-3, Florence, Italy, Sept. 2015.

[38] E.G. Turitsyna and S.K. Turitsyn, "Digital signal processing based on inverse scattering transform," Opt. Lett., vol.38, no.20, pp.41864188, Oct. 2013.

[39] J.E. Prilepsky, S.A. Derevyanko, K.J. Blow, I. Gabitov, and S.K. Turitsyn, "Nonlinear inverse synthesis and eigenvalue division multiplexing in optical fiber channels," Phys. Rev. Lett., vol.113, no.1, pp.013901-1-5, July 2014.

[40] S.K. Turitsyn, "Capacity-achieving techniques in nonlinear channels," The 40th European Conference and Exhibition on Optical Communication (ECOC2014), Paper Mo.4.3.5, Cannes, France, Sept. 2014.

[41] S.T. Le, J.E. Prilepsky, and S.K. Turitsyn, "Nonlinear inverse synthesis for high spectral efficiency transmission in optical fibers," Opt. Exp., vol.22, no.22, pp.26720-26741, Nov. 2014.

[42] H. Bülow, "Experimental assessment of nonlinear Fourier transformation based detection under fiber nonlinearity," The 40th European Conference and Exhibition on Optical Communication (ECOC2014), Paper We.2.3.2, Cannes, France, Sept. 2014.

[43] M.I. Yousefi and F.R. Kschischang, "Information transmission using the nonlinear Fourier transform, part.I: Mathematical tools," IEEE Trans. Inf. Theory, vol.60, no.7, pp.4312-4328, July 2014.

[44] M.I. Yousefi and F.R. Kschischang, "Information transmission using the nonlinear Fourier transform, part.II: Numerical methods," IEEE Trans. Inf. Theory, vol.60, no.7, pp.4329-4345, July 2014.

[45] M.I. Yousefi and F.R. Kschischang, "Information transmission using the nonlinear Fourier transform, part.III: Spectral modulation," IEEE Trans. Inf. Theory, vol.60, no.7, pp.4346-4368, July 2014.

[46] E. Temprana, E. Myslivets, B.P.-P. Kuo, L. Liu, V. Ataie, N. Alic, and S. Radic, "Overcoming Kerr-induced capacity limit in optical fiber transmission," Science, vol.348, no.6242, pp.1445-1448, June 2015

[47] X. Liu, A.R. Chraplyvy, P.J. Winzer, R.W. Tkach, and S. Chandrasekhar, "Phase-conjugated twin waves for communication beyond the Kerr nonlinearity limit," Nature Photonics, vol.7, pp.560568, July 2013.

[48] M. Koga, M. Moroi, and H. Takara, "Optical diversity transmission using WDM signal and phase-conjugate lights through multi-core fiber," Opt. Exp., vol.24, no.9, pp.9340-9352, May 2016.

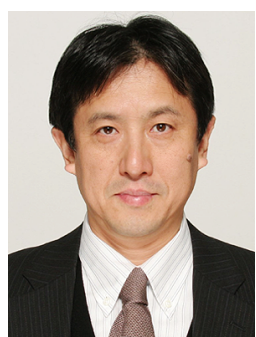

Akihiro Maruta received the B.E., M.E., and Ph.D. degrees from Osaka University, Osaka, Japan, in 1988, 1990, and 1993, respectively, all in communications engineering. He was with the Department of Communications Engineering, Osaka University, in 1993. $\mathrm{He}$ has been an Professor in the Department of Information and Communication Technology, Osaka University, since 2016. His current research interests include optical fiber communication systems and all-optical signal processing. Dr. Maruta is a member of the IEEE Photonics Society and the Optical Society of America. 